\title{
Robo2 drives target selective peripheral nerve regeneration in response to glia derived signals
}

\author{
Patricia L. Murphy ${ }^{1}$, Jesse Isaacman-Beck ${ }^{1}$, and Michael Granato ${ }^{1, \bigotimes}$ \\ ${ }^{1}$ Department of Cell and Developmental Biology, Perelman School of Medicine, University of Pennsylvania, Philadelphia, Pennsylvania, United States of America
}

\begin{abstract}
Peripheral nerves are divided into multiple branches leading to divergent synaptic targets. This poses a remarkable challenge for regenerating axons as they select their original trajectory at nerve branch-points. Despite implications for functional regeneration, the molecular mechanisms underlying target selectivity are not well characterized. Zebrafish motor nerves are composed of a ventral and a dorsal branch that diverge at a choice point, and we have previously shown that regenerating axons faithfully select their original branch and targets. Here we identify Robo2 as a key regulator of target selective regeneration. We demonstrate that Robo2 function in regenerating axons is required and sufficient to drive target selective regeneration, and that Robo2 acts in response to glia located precisely where regenerating axons select the branch-specific trajectory to prevent and correct axonal errors. Combined our results reveal a glia derived mechanism that acts locally via axonal Robo2 to promote target selective regeneration.
\end{abstract}

Keywords: peripheral nerve regeneration, nerve branch, zebrafish, roundabout receptor, robo2, Collagen4a5, target selection

Correspondence: granatom@pennmedicine.upenn.edu

\section{Introduction}

The peripheral nervous system (PNS) has retained a remarkable capacity for axonal regeneration. Following injury, a well characterized injury response system transmits injury signals to the cell body initiating coordinated changes in cellular morphology, gene expression and chromatin remodeling (reviewed in ref. ${ }^{1}$ ). Over the past decades molecular pathways that dramatically increase the rate of regenerative axonal regrowth have been identified ${ }^{2-5}$. Moreover, supplementing pro-regenerative factors to regenerating PNS axons accelerates their regenerative growth rates, yet falls short of providing spatial information to direct axons towards their original, preinjury targets ${ }^{6-9}$. While components of many developmental axon guidance pathways are upregulated after nerve injury, their functional roles in directing regenerating axons are largely unknown (reviewed in ref. ${ }^{10}$ ) Thus, how regenerating axons navigate an environment that differs drastically from an embryonic environment, including how they select their original trajectories when confronted with nerve branch points is not well understood.

In vertebrates, individual peripheral nerves exiting from the spinal cord divide repeatedly into a series of progressively smaller branches, each carrying axons that innervate distinct synaptic targets ${ }^{11}$. Depending on the type and location of a nerve injury, regenerating axons encounter a series of branch-points as they extend toward their origi- nal targets. Thus, for regenerating axons repeatedly selecting their appropriate branch at each branch-point, while a critical step to ensure functional regeneration, represents a formidable challenge. There is considerable evidence that axons have retained some capacity to select their original nerve branch $^{12,13}$, supporting the existence of dedicated molecular mechanisms to govern this process. Although branchspecific regeneration of peripheral axons was first demonstrated over 50 years ago ${ }^{14,15}$, the molecular mechanisms that underly branch-selective, and hence target-selective regeneration are largely unknown. Given the complexity of the process it is maybe not surprising that the regenerative capacity to select the original nerve branch is limited, causing regenerating axons to frequently select inappropriate paths at nerve branch-points 16,17 . For example, motor axons that inappropriately regenerate into nerve branches innervating either the skin or muscles antagonistic to their original target can significantly reduce the level of functional regeneration ${ }^{18,19}$.

We have previously established the optically transparent larval zebrafish as model system to study the cellular and molecular mechanisms of target selective peripheral nerve regeneration in vivo ${ }^{20}$. Zebrafish spinal motor nerves are composed of functionally distinct axonal populations that share a common path before diverging at a defined point into two major branches: a ventral nerve branch consisting of $\sim 50$ individual motor axons that innervate ventral muscle territories, and a dorsal nerve branch consisting of $\sim 20$ motor axons that innervate dorsal muscle territories ${ }^{21-24}$. Following nerve transection regenerating spinal motor axons reliably choose the nerve branch that leads to their original muscle targets ${ }^{12,20}$, and live cell imaging revealed that regenerating axons of the dorsal nerve branch pause at the nerve branchpoint and pause, explore both the incorrect ventral and the original dorsal path before selecting their correct dorsal path. During this exploratory period, a small group of Schwann cells at the nerve branch-point simultaneously upregulates the expression of the extracellular matrix (ECM) component col4a5 and the repulsive axon guidance cue slitla ${ }^{12}$, which have been shown to bind each other with high affinity $^{25}$. Moreover, we previously demonstrated that col4a5 is required during regeneration to guide regenerating dorsal axons ${ }^{12}$, and proposed a model by which Schwann cell-derived Col4a5 scaffolds Slit at the nerve branch-point to prevent regenerating dorsal nerve branch axons from inappropriately entering into and extending along inappropriate trajectories (Fig. 1A-D, ref ${ }^{12}$ ).

To test this model, we first used genetic mutants of the 
Slit-Robo signaling pathway. We find that the Slit-receptor roundabout2 (robo2) and the Slit-Robo co-receptor heparan sulfate (HS) are dispensable for target selection regeneration of ventral nerve axons, but are required to direct regenerating dorsal nerve axons at the branch choice-point. Moreover, we find that robo2 is expressed in dorsal nerve neurons, that forcing robo2 expression in ventral nerve neurons is sufficient to redirect their regenerating axons into the dorsal nerve branch, and that this process requires Col4a5. Finally, using live cell imaging, we demonstrate that during regeneration robo2 exerts its function at the nerve branch-point, preventing and correcting aberrant axonal extension of dorsal nerve axons, thereby promoting growth towards their original, dorsal targets. Combined our results reveal a previously unappreciated role for Slit-Robo signaling in axonal error prevention and correction, critical to ensure fidelity of branch selection and hence target selective regeneration.

\section{Results}

Col4a5 upregulation at the nerve branch-point is critical to guide regenerating dorsal axons. We previously demonstrated that the glycosyltransferase lysyl-hydrosylase3 (lh3) and its substrate collagen-4-alpha-5 (col4a5) are required to direct regenerating dorsal nerve axons towards their original targets ${ }^{12}$. Lh3 is constitutively expressed at low levels and acts in Schwann cells to promote target selective regeneration, while col4a5 expression is transiently upregulated 8-15 hours post transection (hpt) in a small subset of Schwann cells near the nerve branch-point (Fig. 1C and ref. ${ }^{12}$, suggesting that col4a5 expression restricted to the nerve branch-point might be instructive in directing regenerating axons. To test this idea, we generated a transgenic line, $T g(\operatorname{sox} 10: \operatorname{col} 4 a 5-M y c)$, in which col4a5 is now expressed in all Schwann cells, prior to and following peripheral nerve transection ${ }^{12}$. Prior to nerve transection, dorsal nerves in wildtype siblings appear indistinguishable from those in transgenic animals expressing col4a 5 in all Schwann cells (compare Fig.1E to H). Specifically, we quantified targeting of $T g(i s l 1: G F P)^{+}$dorsal nerve axons prior to nerve transection in 5 day old animals. Dorsal nerve axons tightly fasciculate with one another, precluding us from quantifying individual axons contained in $T g(i s l 1: G F P)^{+}$dorsal nerves. We therefore quantified the number of discernable $T g(i s l 1: G F P)^{+}$fascicles and determined the fraction of fascicles within the dorsal muscle target area, which we previously defined as spanning $30^{\circ}$ prior to transection (dorsal ROI Fig. $1 \mathrm{D} ;{ }^{12}$ ). When we compared these fractions across genotypes, we found no significant difference in dorsal fascicles between $T g(\operatorname{sox} 10:$ col4a5-Myc) animals and wildtype siblings (Table S1), suggesting that in $T g(\operatorname{sox} 10:$ col4a5-Myc) animals, dorsal nerve targeting during development is unaffected.

To determine whether spatially restricted expression of col4a5 is critical for target selective regeneration, we laser transected dorsal nerves in wildtype and $T g$ (sox 10:col4a5Myc) larvae and compared target selective regeneration at 48 hpt, when wildtype motor axons have re-established func- tional connections with their muscle targets ${ }^{20}$. In wild type animals, $70 \%$ of fascicles containing dorsal nerve axons regenerated to their original dorsal target area (Fig. 1F, G; 37 nerves from 11 larvae), consistent with previous findings that regenerating axons readily select their original branch and targets ${ }^{12}$. In contrast, transgenic expression of col4a 5 in all Schwann cells reduced target selective regeneration significantly. In $T g$ (sox10:col4a5-Myc)-expressing larvae, only $46 \%$ of the fascicles containing dorsal nerve axons selected their original dorsal trajectory (40 nerves from 11 larvae), concomitant with an 1.8 fold increase from $30 \%$ to $54 \%$ fascicles selecting incorrect ventral and lateral trajectories (Fig. 1I-J; Table S1; $p=0.0002$, Fischer's exact test). We next examined the regeneration error rate for individual nerves at $48 \mathrm{hpt}$ and found that, when compared to wildtype siblings, nerves in $T g$ (sox10:col4a5-Myc)-expressing larvae displayed errors at a significantly higher rate (Fig. $1 \mathrm{~K} ; \mathrm{p}=0.0001$, one-tailed t-test). Thus, expanding col4a5 expression from a small subset of Schwann cells strategically positioned at the nerve branch region to all Schwann cells impairs target selective regeneration. Moreover, the resulting phenotype closely mirrors the phenotype in mutants lacking col4a $5^{12}$. These results support the idea that col4a5's transient expression in a subset of Schwann cells at the nerve branch point where regenerating axons of the dorsal branch select their branch specific trajectory is of functional importance.

\section{Slit-Robo signaling is required for target selective re-} generation. The same small group of Schwann cells that upregulates col4a5 post injury concurrently upregulates slit1a expression ${ }^{12}$, suggesting that similar to col4a5, slitla might play a functional role in target selective regeneration. Slitla encodes a canonical ligand for the Roundabout (Robo) family of repulsive axon guidance receptors ${ }^{26}$, and is the only one of the four Slit ligands whose injury induced expression mirrors that of $c o l 4 a 5^{12}$. Moreover, in the developing zebrafish visual system, Col4a5 directly binds to and is required for basement membrane anchoring of Slit, which guides laminar targeting of retinal ganglion cell axons through robo $2^{25}$. We therefore wondered whether robo2 is involved in guiding regenerating dorsal axons. Using whole mount fluorescent in situ hybridization (ISH), we detected robo2 mRNA expression in $T g(i s l 1: G F P)^{+}$motor neurons of the dorsal nerve prior to transection and also during regeneration (Fig. S1).

We next asked whether Slit-Robo signaling plays a functional role in target selective regeneration. For this we examined dorsal nerve regeneration in genetic mutants for two Slit-Robo signaling components: mutants for exotosin-like-3 (extl3), which lack Heparan sulfate (HS) ${ }^{27}$, a glycosaminoglycan critical to stabilize Slit-Robo binding ${ }^{28}$, and mutants for the Robo-receptor roundabout2 (robo2). We first examined dorsal nerve regeneration in extl3 mutants, which at 5 dpf lack detectable levels of $\mathrm{HS}^{27}$. Prior to nerve transection, targeting of dorsal nerve axons in $5 \mathrm{dpf}$ day old extl3 mutants was indistinguishable from their siblings (compare Fig. 2A to D; quantified in Table S1). In wildtype siblings, $68 \%$ of regenerating dorsal nerve fascicles returned to their original, dorsal targets (Fig. 2B-C), while 32\% selected ven- 
bioRxiv preprint doi: https://doi.org/10.1101/2020.12.07.406942; this version posted December 7, 2020. The copyright holder for this preprint (which was not certified by peer review) is the author/funder. All rights reserved. No reuse allowed without permission.
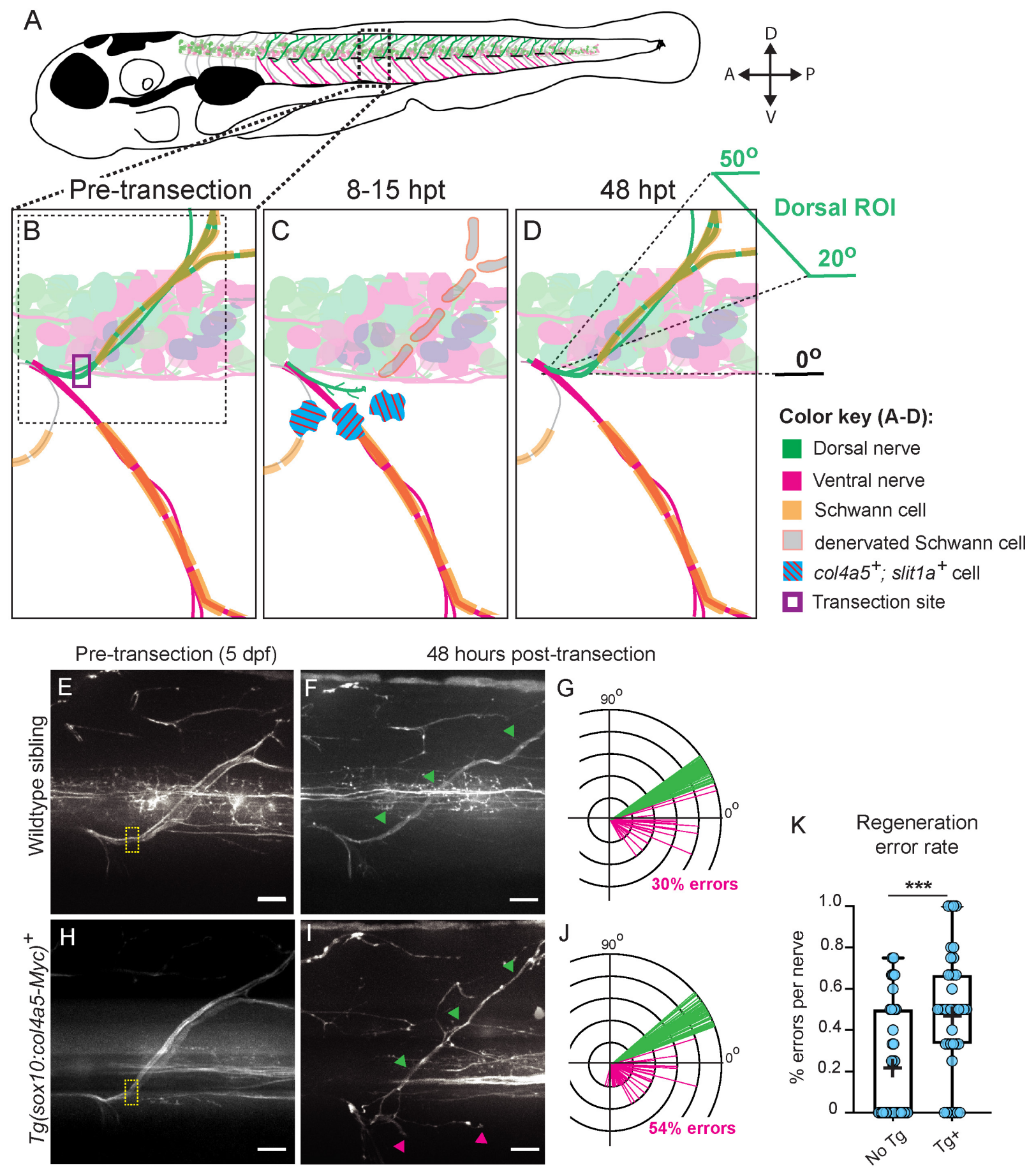
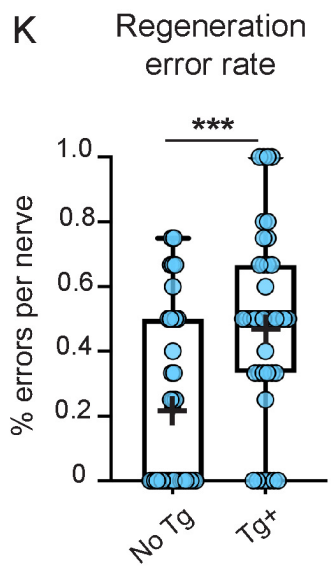

Fig. 1. Spatiotemporal restriction of col4a5 to the nerve branch-point is required for target-selective axon regeneration. Top: In 5 dpf larval zebrafish (schematized in A), dorsal (green) and ventral (magenta) motor nerves exit the spinal cord in each body hemisegment via the ventral motor exit point (MEP) and diverge at a stereotyped branch-point to innervate the dorsal and ventral muscles, respectively. (B) Magnified schematic of single motor nerve in dashed box in (A) pre-transection, showing dorsal (green) and ventral (magenta) branches of the spinal motor nerve and associated Schwann cells (orange). Purple box, transection site. (C) At 8-15 hours post transection (hpt), growth cones enter the transection gap, and a small subset of Schwann cells at the nerve branch-point upregulate col4a5 and slit1a (blue with red stripes). (D) By 48hpt, the majority of dorsal axons regenerate into the dorsal ROI, defined as $20-50^{\circ}$ with respect to spinal cord. Bottom: Representative images of $T g\left(i s / 1\right.$ :GFP) ${ }^{+}$dorsal nerves at $5 \mathrm{dpf}$ and $48 \mathrm{hpt}$, respectively in wildtype siblings (E, F) and $\mathrm{Tg}($ sox 10:col4a5-Myc) larvae (H,I). Yellow boxes, transection sites; green arrowheads, dorsal regrowth; magenta arrowheads, "errors" that regrew along non-dorsal paths. Scale bars, 10um. Sholl diagrams showing overlay of all Tg(is/1:GFP) + fascicle trajectories at 48 hpt in wildtype siblings (G, $n=96$ fascicles) and $T g$ (sox10:col4a5-Myc) larvae ( $\mathbf{J}, \mathrm{n}=133$ fascicles). For wildtype siblings, fascicles were counted for $\mathrm{n}=37$ nerves in 11 larvae; for $\mathrm{Tg}($ sox 10:col4a5-Myc) larvae, fascicles were counted for $n=40$ nerves in 11 larvae. Green lines represent fascicles inside dorsal ROI; magenta lines represent fascicles outside of the dorsal ROI. Proportion of fascicles inside the dorsal ROI at $48 \mathrm{hpt}$ was compared between $\mathrm{Tg}$ (sox 10:col4a5-Myc) larvae and wildtype siblings using one-tailed Fischer's exact test, $(\mathrm{p}=0.0002)$. (K) Graph of regeneration error rate for wildtype siblings ( $\mathrm{No} \mathrm{Tg})$ and $\mathrm{Tg}(\mathrm{sox} 10: \mathrm{col} / 4 \mathrm{a}-\mathrm{Myc})$ larvae $\left(\mathrm{Tg}{ }^{+}\right.$) compared using one-tailed t-test $(p=0.0001)$. Each dot represents error rate for one nerve; plus sign marks the mean; ${ }^{* *}$ denotes $p<0.001$. 
tral and ventro-lateral trajectories (38 nerves from 19 larvae). In contrast, in extl 3 mutants we observed a 1.6 fold increase (from $32 \%$ to $52 \%$ ) of dorsal nerve fascicles failing to select their dorsal trajectory, instead extending along erroneous ventral or ventro-lateral trajectories (23 nerves from 7 larvae) (Fig. 2E-F; Table S2, p = 0.0105, Fischer's exact test). Similarly, when compared to wildtype siblings, individual nerves in extl 3 mutants formed errors at a significantly higher rate at 48 hpt (Fig. 2G; p = 0.003, one-tailed t-test).

We next examined the role of robo2 in dorsal nerve regeneration. Prior to transection, dorsal nerve targeting in robo2 mutant animals was slightly lower than what we observed in robo2 wildtype siblings (compare Fig. $2 \mathrm{H}$ to $\mathrm{K}$; quantified in Table S1), yet still within the range we have previously observed in wild type animals ${ }^{12}$. Following nerve transection in wild type siblings, $34 \%$ of dorsal nerve fascicles failed to select their original trajectory, while the vast majority returned to their original target area (66\%; Fig. 2IJ). In contrast, in robo 2 mutants only $50 \%$ of regenerating dorsal nerve fascicles returned to their original target area, an almost 1.5 fold increase of $T g(i s l 1: G F P)^{+}$fascicles now extending along aberrant ventral or ventro-lateral trajectories (Fig. 2L-M; Table S2, p = 0.0134, Fischer's exact test). Similarly, when compared to nerves in wildtype siblings at $48 \mathrm{hpt}$, individual nerves in robo 2 mutants exhibited significantly higher error rates (Fig. $2 \mathrm{~N} ; \mathrm{p}=0.0116$, one-tailed t-test). Together these results demonstrate that Slit-Robo signaling plays a functional role in directing regenerating dorsal nerve axons along their original, pre-injury trajectories. Finally, to determine whether extl3 and robo2 play a selective role in promoting target selection of dorsal, rather than ventral nerve axons, we transected ventral nerves in extl3 and robo 2 mutants. At $48 \mathrm{hpt}$, ventral nerves in extl3 and robo 2 mutants are indistinguishable from their siblings (Fig. S2, demonstrating that Slit-Robo signaling is selectively required for dorsal nerve target selective regeneration.

\section{Robo2 promotes target selective regeneration at the nerve branch-point by preventing and correcting er-} rors. To further understand the cellular mechanisms by which robo2 promotes target selective regeneration, we examined the dynamics of regenerating axons navigating the nerve branch-point in robo2 mutants. We have previously shown that after dorsal nerve transection in wildtype larvae, regenerating axons pause at the nerve branch-point and extend growth cones towards their original dorsal targets, as well as along erroneous ventral and lateral trajectories. Over the next few hours erroneous projections are destabilized, while growth cones along the dorsal path stabilize and continue to extend towards their original targets ${ }^{12}$. To determine whether robo2 directs regenerating dorsal nerve axons early in the process by minimizing the formation of erroneous projections, or subsequently by destabilizing already extending erroneous projections, we performed time-lapse imaging between 8 and $20 \mathrm{hpt}$ as regenerating robo 2 mutant dorsal nerve axons navigate the branch choice-point (Figure 3, Movies S1 and S2). From these movies, we quantified the number of erroneous projections (errors), defined as
$T g(i s l 1: G F P)^{+}$growth $\geq 1 \mu \mathrm{m}$ that extended from the nerve branch-point along erroneous ventral or lateral trajectories (see Methods for more details). When compared to wild type siblings, robo2 mutants exhibit no significant difference in the number of errors that form at the branch-point (Fig. S3). To determine whether there was a deficit in error correction at the branch-point in robo2 mutants, we counted the number of errors (magenta arrowheads, Fig. 3, Movies S1 and S2) that were corrected. Errors were counted as "corrected" if they retracted within $<1 \mu \mathrm{m}$ away from the nerve branchpoint. When compared to siblings, robo 2 mutants displayed a significant decrease in the percent of errors that were corrected during early regeneration (Figure 3I).

Based upon the well characterized role of Slit-Robo in axon repulsion ${ }^{26}$, we considered whether in robo 2 mutants the deficit in error correction at the nerve branch-point might be due to reduced error retraction. To test this, we measured in wildtype siblings and robo2 mutants the percent of time that errors spent retracting, extending or being stable (no movement). We failed to detect any significant difference in relative time that errors spent retracting in robo2 mutants compared to wild type siblings (Fig. 3J). Similarly, we did not observe any differences in the average speed of extension or retraction in robo 2 mutants when compared to siblings (Fig. S3B). Instead, compared to wildtype siblings, erroneous projections (errors) in robo2 mutants spent significantly more time extending (Fig. 3J). This is the result of a combined reduction in the time that errors spent retracting and stable in robo 2 mutants, neither of which is statistically significant on its own (Fig. 3J). These results suggest that rather than promoting axonal retraction along incorrect trajectories, robo 2 promotes dorsal nerve target selection by preventing axon extension along erroneous ventral and lateral trajectories. Consistent with this idea, when compared to wild type siblings, erroneous projections in robo2 mutants grew longer distances (Fig. 3K). Combined with previous results this provides strong support for a model in which robo2 expression on regenerating dorsal prevents and corrects errors at the nerve branch-point in response to Slit1a transiently produced by a small subset of adjacent Schwann cells and spatially scaffolded by Col4a5. By preventing error extensions, robo 2 tilts the balance between extension and retraction such that errors retract more often than they extend. This results in shorter errors that are readily corrected through robo2-independent mechanisms of retraction, ultimately biasing regenerating dorsal nerve axons towards their original dorsal trajectory.

Robo2 expression drives target selective regeneration. We next asked how Slit-Robo signaling selectively influences regeneration of dorsal but not ventral nerve axons. One possibility is that robo2 functions selectively in dorsal nerve axons, enabling regenerating axons of only the dorsal but not the ventral branch to mount a slitla-dependent error response at the nerve branch-point. We hypothesized that if this were the case, forcing robo 2 expression in regenerating ventral nerve axons would redirect them onto a dorsal trajectory. To test if robo 2 expression is indeed suffi- 
bioRxiv preprint doi: https://doi.org/10.1101/2020.12.07.406942; this version posted December 7, 2020. The copyright holder for this preprint (which was not certified by peer review) is the author/funder. All rights reserved. No reuse allowed without permission.
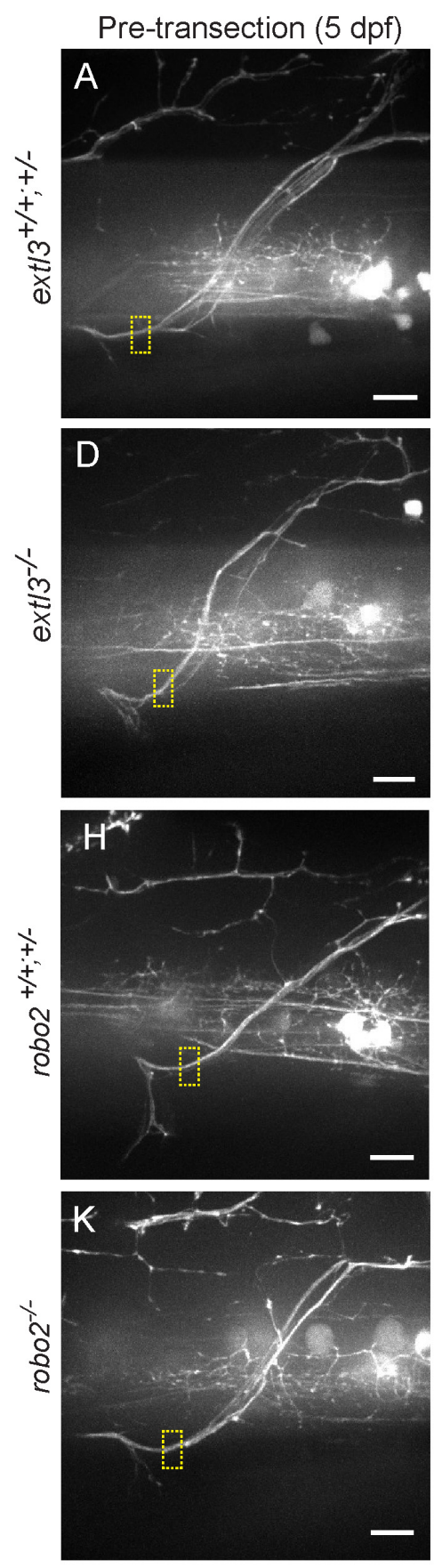
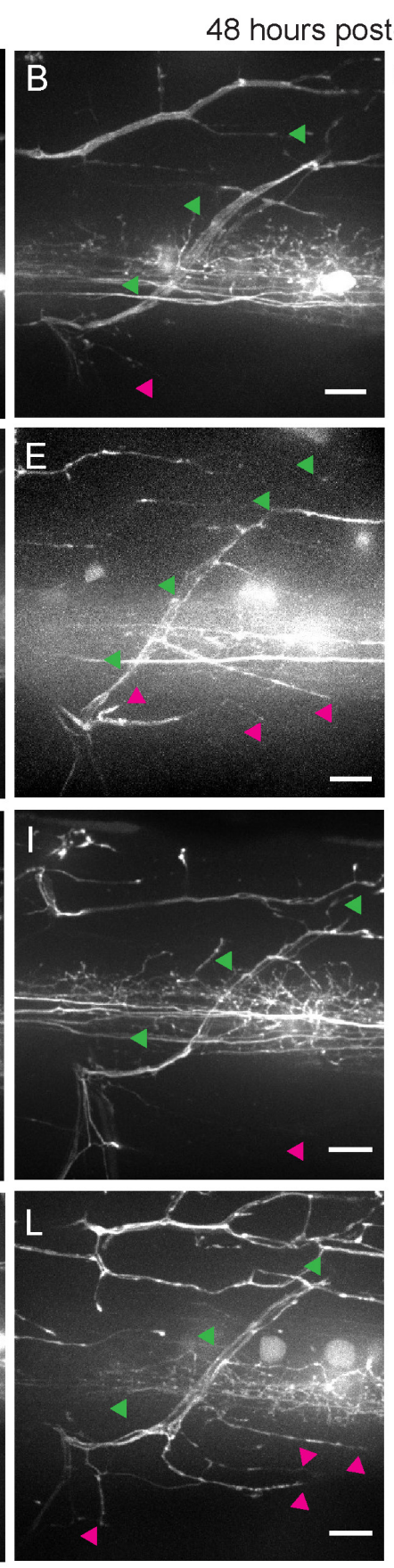
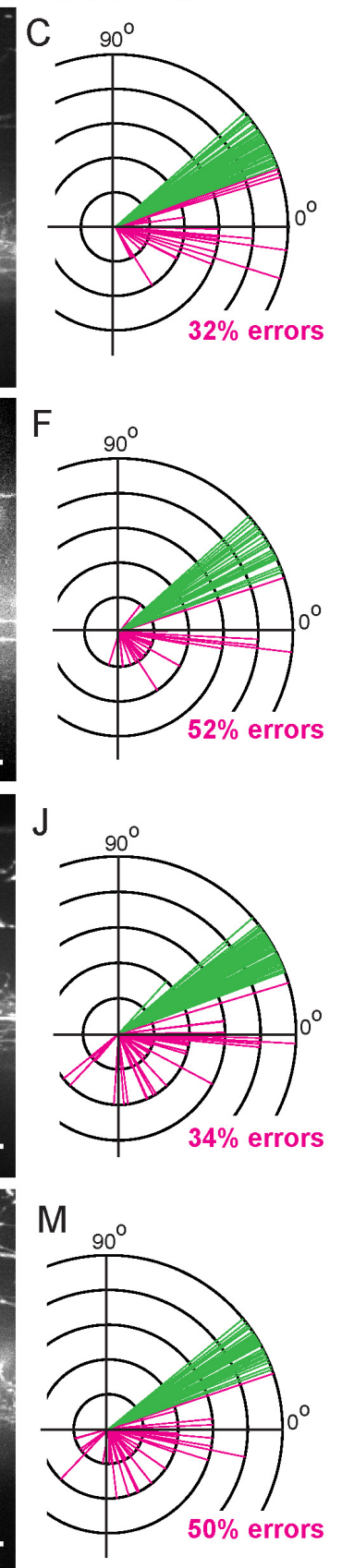

\section{G Regeneration error rate}

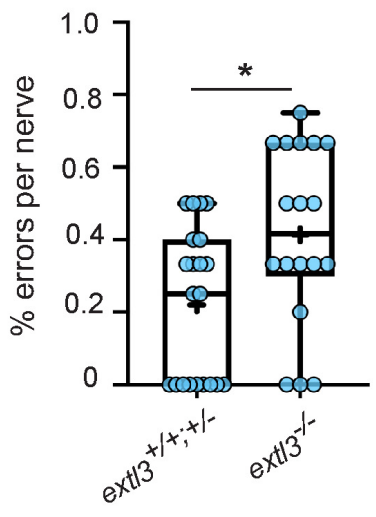

\section{$\mathrm{N}$ \\ Regeneration error rate}

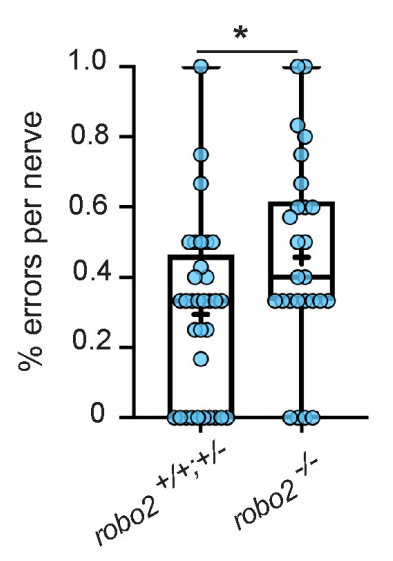

Fig. 2. Slit-Robo signaling is required to guide regenerating dorsal nerve branch axons. Representative images of dorsal nerves in ext/3 wildtype siblings at 5 dpf and $48 \mathrm{hpt}$, respectively in ext/3 wildtype siblings $(\mathbf{A}, \mathbf{B})$, ext/3 ${ }^{-/-}$(D, E), robo2 wildtype siblings $(\mathbf{H}, \mathbf{I})$, robo2 ${ }^{-/-}(\mathbf{K}, \mathbf{L})$. Yellow boxes, transection sites; green arrowheads, dorsal regrowth; magenta arrowheads, "errors" that regrew along ventral and ventro-lateral paths. Scale bars, 10um. Sholl diagrams showing overlay of all Tg(is/1:GFP) fascicle trajectories at $48 \mathrm{hpt}$ in ext/3 wildtype siblings $(\mathbf{C}, \mathrm{n}=98$ fascicles $)$, ext/3 ${ }^{-/-}(\mathbf{F}, \mathrm{n}=58$ fascicles$)$, robo2 wildtype siblings $(\mathbf{J}, \mathrm{n}=108$ fascicles $)$, robo2 ${ }^{-/-}$(M) $\mathrm{n}=97$ fascicles). Green lines represent fascicles inside dorsal ROI; magenta lines represent fascicles outside of the dorsal ROI. For ext/3 wildtype siblings, fascicles were counted in $n=38$ nerves in 19 larvae; for ext/3 $3^{-/}$larvae, fascicles were counted in $n=23$ nerves in 7 larvae; for robo2 wildtype siblings, fascicles were counted in $n=33$ nerves in 16 larvae; for robo2 $-/-$ larvae, fascicles were counted in $n=26$ nerves in 9 larvae. Proportion of fascicles inside the dorsal ROI at 48 hpt was compared using one-tailed Fischer's exact test between ext/3 wildtype siblings and ext/3 ${ }^{-/-}$larvae $(p=0.0105)$ and robo2 wildtype siblings and robo2 ${ }^{-/-}$larvae $(p=0.0134)$. $(G)$ Graph of regeneration error rate for ext/3 wildtype siblings and ext/3-/- larvae compared using one-tailed t-test $(p=0.003)$. (N) Graph of regeneration error rate for robo2 wildtype siblings and robo2 ${ }^{-/-}$larvae compared using one-tailed t-test $(p=0.0116)$. Each dot represents error rate for one nerve. ${ }^{*}$ denotes $p<0.05$.

cient to drive target selective regeneration, we used the motor neuron-specific $\mathrm{mnx} 1$ promotor $^{29}$ to transiently express
mKate alone or mKate with robo2 in small subsets of motor neurons (for more details see Methods). Importantly, when 

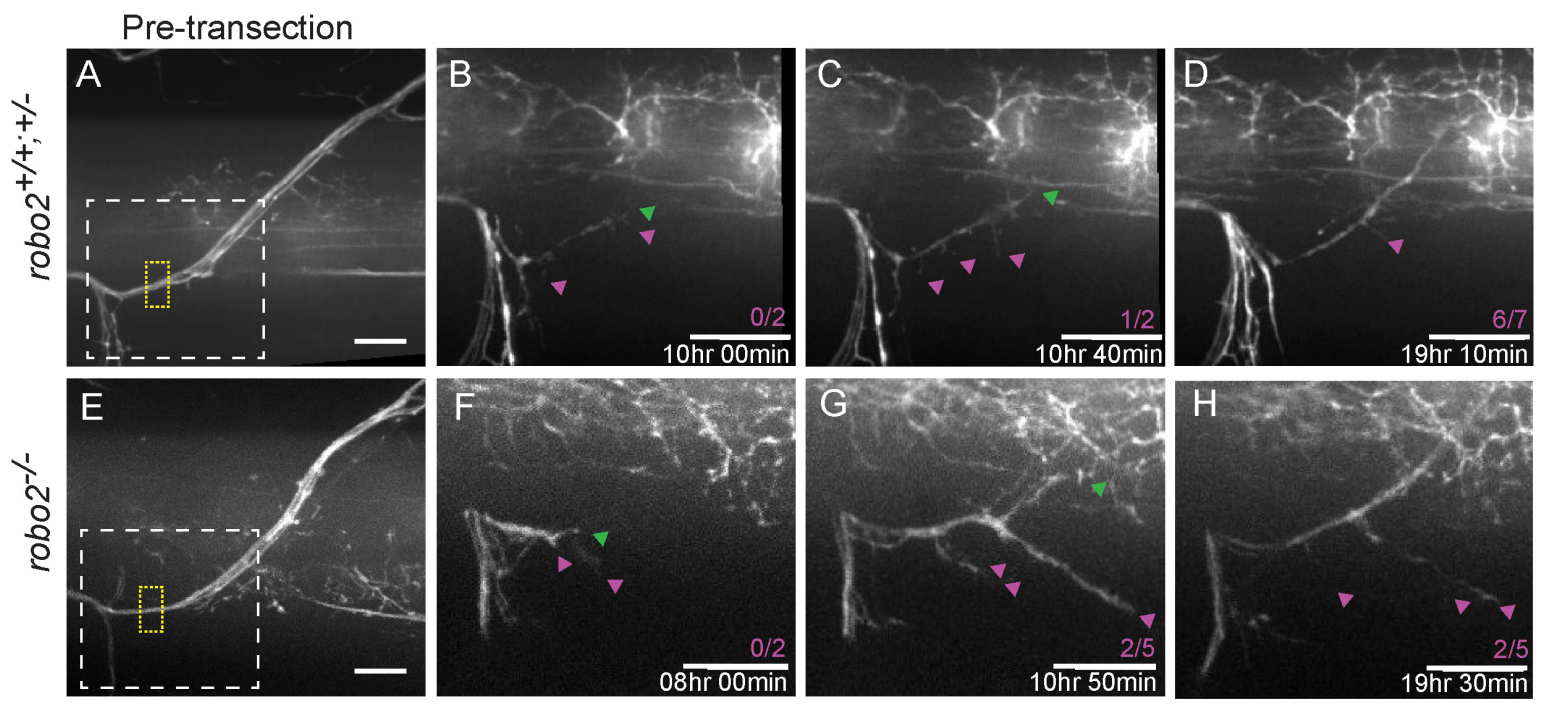

I Errors corrected (8-20hpt)

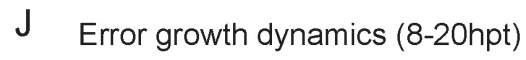

K Max. error length
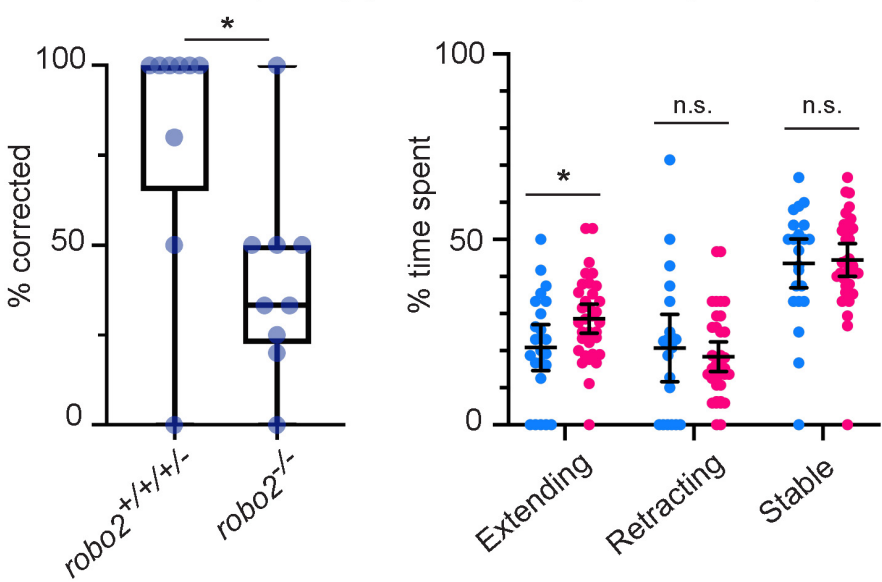

- robo $2^{+/+++/-}$

- robo $2^{-/-}$

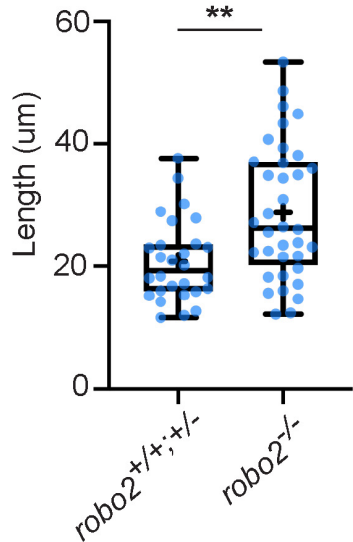

Fig. 3. robo2 prevents error extension at the nerve branch-point during regeneration. Representative images of $T g$ (is/1:GFP) nerves in robo2 ${ }^{+/-}$(wildtype sibling) (A) robo2 $^{-/-}(\mathbf{E})$ at $5 \mathrm{dpf}$ and during regeneration (B-D, F-H). Dashed yellow box, transection site; dashed white box, area enlarged $2 \mathrm{x}$ in B-D and F-H; green arrowheads mark dorsal regrowth; magenta arrowheads mark errors. Time post transection is denoted by white text in lower right of B-D and F-H. Errors are counted in magenta text in lower right of B-D and F-H as a fraction of [errors formed/errors corrected]. Scale bars, 10um. (I) Percent of errors corrected 8-20 hpt in wildtype siblings (robo2 $2^{+/+;+/-}, \mathrm{n}=9$ nerves) and robo2 ${ }^{-/-}$larvae $(n=10$ nerves). Errors were counted as "corrected" when their length measured from the nerve branch-point was $<1$ um. Each dot represents one nerve. Ranks were compared between genotypes using two-tailed Mann Whitney test $(p=0.0208)$. (J) Quantification of regenerating axon dynamics in wildtype siblings and robo2 $^{-1-}$ larvae 8-20 hpt plotted by total time spent extending, retracting, and stable (no movement). Each dot represents one error (for siblings, $\mathrm{n}=23 \mathrm{errors}$; for robo2 ${ }^{-/}, \mathrm{n}=34$ errors). Error movements were examined in $10 \mathrm{~min}$ intervals and classified as extensions when there was a 1 um increase in error length measured from the motor exit point (MEP); movements were classified as retractions when $\geq 1$ um decrease in error length measured from the MEP; errors were classified as stable when no movement $\geq 1$ um occurred. Line, mean; error bars, $95 \%$ confidence intervals. Means were compared between genotypes using two-tailed t-tests (extending, $p=0.0365$; retracting $p=0.9131$; stable, $p=0.2491)$. (K) Maximum length of errors in wildtype siblings and robo2 $2^{-/}$larvae in ums measured from the motor exit point (MEP). Each dot represents one error. Plus sign marks the mean. Means were compared between genotypes using two-tailed t-test $(p=0.0012)$. ${ }^{* *}$ denotes $p<0.01 ;{ }^{*}$ denotes $p<$ 0.05 ; n.s. denotes "not significant."

compared to mKate expression, robo2-mKate expression in motor neurons did not impair their ability to grow nor did it change their developmental bias in selecting a ventral or dorsal trajectory (Fig. S4). This is consistent with the absence of a developmental motor axon phenotype in robo2 mutants (Table S1), further confirming that robo2 acts selectivity during the regeneration process. To determine whether robo 2 is sufficient to promote dorsal branch selection in regenerating axons, we screened for spinal motor nerves with small subsets of mKate ${ }^{+}$axons along the ventral, but not the dorsal branch (Fig. 4A and 4C). We laser transected these ventral nerves and assessed the regeneration of $\mathrm{mKate}^{+}$fascicles at $48 \mathrm{hpt}$. We found that regenerating $\mathrm{mKate}^{+}$ventral nerve axons always selected a ventral path towards their original targets (Fig. 5B; Table S3, $\mathrm{n}=13 / 13$ nerves), consistent with previous results ${ }^{20}$. In contrast, forcing robo2-mKate expression in regenerating ventral nerve axons was sufficient to redirect them onto a dorsal trajectory (Fig. 4C-D; Table S3, $\mathrm{n}=7 / 15$ nerves, Fischer's exact test $\mathrm{p}=0.0069$ ). Importantly, the trajectories taken by these axons were indistinguishable 

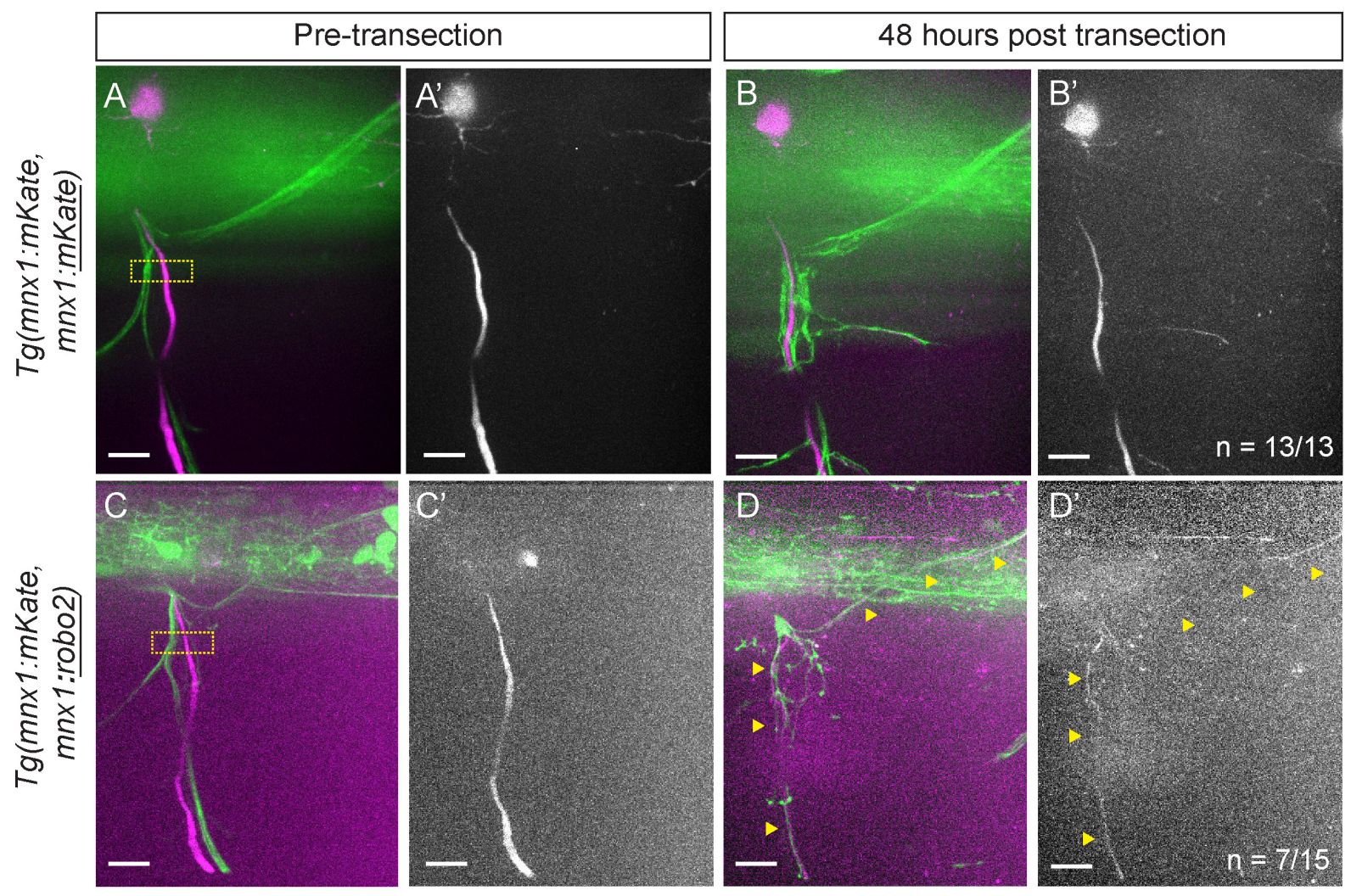

Fig. 4. robo2 is sufficient to promote dorsal branch-selection by regenerating axons. Representative images of $T g(h b 9: G F P)$ (green) nerves with small numbers of fascicles expressing transient $\operatorname{Tg}(m n \times 1: m K a t e, m n x 1: m K a t e)(\mathbf{A}-\mathbf{B})$ or $\operatorname{Tg}(m n \times 1: m K a t e, m n x 1: r o b o 2)(\mathbf{C}-\mathbf{D})$ (magenta) in small subsets of ventrally projecting motor axons. Merged GFP and mKate images shown at $5 \mathrm{dpf}(\mathrm{A}, \mathrm{C})$ and $48 \mathrm{hpt}(\mathrm{B}, \mathrm{D})$. mKate channel is shown alone at $5 \mathrm{dpf}\left(\mathbf{A}^{\prime}, \mathbf{C}^{\prime}\right)$ and $48 \mathrm{hpt}\left(\mathbf{B}^{\prime}, \mathbf{D}^{\prime}\right)$. In larvae expressing $\mathrm{Tg}(\mathrm{mnx} 1$ :mKate, $m n x 1: m K a t e), n=13 / 13$ nerves had only ventral regrowth of mKate ${ }^{+}$fascicles, like in the example shown. In larvae expressing $T g(m n x 1: m K a t e, ~ m n x 1: r o b o 2), n=7 / 15$ nerves had dorsal regrowth of mKate ${ }^{+}$fascicles, like in the example shown. Proportions of dorsal regrowth were compared between conditions using one-tailed Fischer's exact test $(p=0.0054)$. Images were processed as described in Materials and Methods. Dashed yellow boxes, transection site. Scale bars, $10 u m$.

from those taken endogenously by dorsal nerve axons (compare to Fig. 2B, I). Thus, robo2 is both required and sufficient to drive target selective regeneration.

\section{Robo2 requires col4a5 function for target selective re-} generation. In robo2 and col4a5 mutants, ventral branch axons reliably regenerate along their appropriate ventral path (Fig. 4; ref. ${ }^{12}$ ), while dorsal branch axons frequently fail to select their original dorsal trajectory and instead extend along erroneous, ventral and lateral trajectories. Because of the similarities of their mutant phenotypes, we next asked whether robo 2 and col4a5 act through two distinct pathways or whether they are both part of one common pathway. We reasoned that if the latter was the case, then redirecting ventral nerve axons towards dorsal trajectories via forced robo 2 expression should depend on col4a5 function. To test this hypothesis, we repeated the robo 2 mis-expression experiment driving sparse expression of either mKate or robo2-mKate in small subsets of regenerating ventral nerve axons, but now in a col4a5 mutant background. Prior to nerve transection at $5 \mathrm{dpf}$, there was no difference between the branch-selection of sparsely labeled wildtype and robo2-expressing axons in col4a5 siblings or mutants (Fig. S5). This is consistent with our previous findings that col4a5 is dispensable for spinal motor nerve development ${ }^{12}$. Like before, we selected spinal motor nerves with small subsets of mKate ${ }^{+}$axons along the ventral, but not the dorsal nerve branch, laser transected these ventral nerves and at $48 \mathrm{hpt}$ assessed the regeneration of $\mathrm{mKate}^{+}$axons. In col4a5 siblings and mutants, regenerating ventral nerve axons expressing mKate faithfully selected their original, ventral trajectory (Fig. 5A-B; Fig. S6A-B; Table S3, col4a5 siblings: $\mathrm{n}=12 / 12$; col4a5 mutants $\mathrm{n}=8 / 8$ ). In col4a5 siblings, robo2-mKate expression was sufficient to redirect regenerating ventral nerve axons onto a dorsal trajectory (Fig. S6C-D; Table S3, $\mathrm{n}=5 / 14$ nerves, Fischer's exact test $\mathrm{p}=0.0425$ ). In contrast, in col4a5 mutants, robo2mKate expression failed to redirect ventral nerve axons onto a dorsal trajectory (Fig. 5C-D; Table S3, $\mathrm{n}=0 / 9$ ), demonstrating that col4a5 function is required for robo2 to redirect ventral nerve axons dorsally. This provides compelling evidence that col4a5 and Slit-Robo act in a common genetic pathway that promotes dorsal branch selection of regenerating axons. Combined, our results support a model in which a small subset of Schwann cells strategically located at the branch choice point upregulate the expression of colta5 and slitla in response to nerve injury. As regenerating axons approach the branch choice point, Robo2 function selectively in dorsal nerve axons prevents and correct erroneous projections 
along ventral and lateral trajectories, thereby biasing axonal regrowth towards their original, dorsal trajectory (Fig. 6).

\section{Discussion}

In response to peripheral nerve injury, regenerating axons face the challenge of navigating toward and reconnecting with their original synaptic targets. The difficulty of this task increases with the architectural complexity of the injured nerve. After exiting the spinal cord, peripheral nerves repeatedly divide into progressively smaller branches, frequently leading to different targets ${ }^{11}$. Regenerating axons may therefore encounter multiple nerve branch-points where they confront the choice to select their appropriate, preinjury trajectory. This task is further compounded by an environment that is dramatically different from the one they successfully navigated during development. Despite constituting an enormous navigational challenge, regenerating axons are able to preferentially select their original nerve branch ${ }^{12,15,30}$, and to regrow toward appropriate targets ${ }^{31-34}$. Combined, previous studies strongly support the notion that regenerating axons are guided at nerve branch-points by dedicated molecular mechanisms, yet few such mechanisms have been described. Here we identify a molecular pathway critical for communication between glia cells located at a nerve branch-point and regenerating axons to direct axons of one nerve branch onto their preinjury trajectory. Specifically, our results provide compelling evidence for transient, spatially restricted, and tightly coordinated signaling events between col4a5/slitlaexpressing Schwann cells and robo2-expressing regenerating axons at the nerve branch-point critical to promote target selective regeneration.

Robo2 selectively destabilizes erroneous axonal extension at the nerve branch-point. Live cell imaging experiments provide compelling evidence for a Robo2dependent mechanism that directs regenerating axons into the appropriate nerve branch as they encounter the branch choice-point. As they encounter the nerve branch-point, regenerating dorsal nerve axons in both wildtype and robo2 mutants initiate growth $(\sim 1 \mu \mathrm{m})$ along erroneous ventral or ventro-lateral trajectories with similar frequencies (Fig. $\mathrm{S} 3 \mathrm{~A})$. In contrast to wildtype, robo2 mutant axons along these erroneous trajectories are significantly more likely to extend (Fig. 3J), ultimately growing for distances 1.5 to 3 times longer than those in the wildtype (Fig. 3K). Thus, rather than preventing initial error formation per se, robo2 destabilizes erroneous axons $(>\sim 1 \mu \mathrm{m})$, thereby preventing their further growth (Fig. 3K). Thus, while robo2 destabilizes erroneous axonal growth, it promotes rather than inhibits regeneration. This is markedly different from the role that canonically repulsive axon guidance systems, including Slit-Robo, often play in inhibiting axon extension after injury, resulting in poor or stalled regeneration ${ }^{10,35}$. For example, in C. elegans, Slit (slt-1) and Robo (sax-3) inhibit the extension of the mechanosensory PLM axon after transection, ultimately leading to reduced regeneration ${ }^{36}$. Rather than negatively impacting axon regeneration, we find that growth rates of regenerating axon outgrowth in robo $2 \mathrm{mu}-$ tants are identical to those in wild type animals (Fig. S3B). This provides strong evidence that for zebrafish peripheral nerve regeneration robo2-independent mechanisms promote axon outgrowth, while robo2's role is to selectively bias regenerative growth of dorsal nerve axons toward their original trajectory.

How similar is this robo2-dependent mechanism to other, well documented mechanisms known to promote target selective regeneration in mammals? It is well documented that in mammals motor axons preferentially regenerate into their original nerve branches ${ }^{13-15}$, and that this process is regulated by Schwann cells and by nerve end organs such as muscle and skin ${ }^{37,38}$. For example, after injury, Schwann cells in the distal stump upregulate branch-specific neurotrophic factors and cell adhesion molecules ${ }^{39-42}$. These molecules support the outgrowth and maintenance of appropriate axonal populations ${ }^{43,44}$ such that when axons regenerate into inappropriate nerve branches, the resulting errors are pruned away over weeks or months ${ }^{30,45}$. Thus, in contrast to well documented pruning mechanisms occurring long after axons have regenerated towards incorrect targets, the robo2-dependent mechanism we describe here is engaged during the time period when regenerating axons are confronted at a choice point with the task to select their original trajectory, thereby promoting target selective regeneration locally and on a much shorter timescale.

\section{A Co4a5/glia Robo2/axon dependent mechanism pro- vides local guidance to promote target selective re-} generation. In response to peripheral nerve injury, Schwann cells distal to the injury site dedifferentiate through a well characterized molecular pathway ${ }^{46,47}$. These dedifferentiated Schwann cells exhibit differences in gene expres$\operatorname{sion}^{39-41}$, yet the functional significance of these gene expression differences, including their functional roles in branch selection and thus target selective regeneration have remained largely unknown. We previously reported that in response to peripheral nerve injury in zebrafish, a small, selective group of Schwann cells $(\sim 1-3)$ strategically located at where dorsal nerve branch deviates from the ventral nerve branch, upregulate both col4a5 and its binding partner, the repulsive guidance cue Slit $1 a^{12,25}$. While we had shown that col4a5 is critical for target selective regeneration, whether the post injury expression of col4a5 spatially restricted to just a few Schwann cells was important for target selectivity was unclear. Similarly, whether Slit1a played a functional role in this process and whether Slit1a and col4a5 expression were functionally related had not been defined.

Our results demonstrate that expanding the expression of col4a5 to all Schwann cells severely impacts target selective regeneration (Fig. 1). Importantly, rather than extending along random trajectories, regenerating dorsal nerve axons now extended along erroneous ventral and ventro-lateral trajectory similar to those we observe in mutants lacking col4a5, robo 2 and extl3, respectively (Fig. 2; ref. ${ }^{12}$ ). Moreover, in animals expressing col4a5 in all Schwann cells, regenerating dorsal nerve axons made errors precisely where they pause 


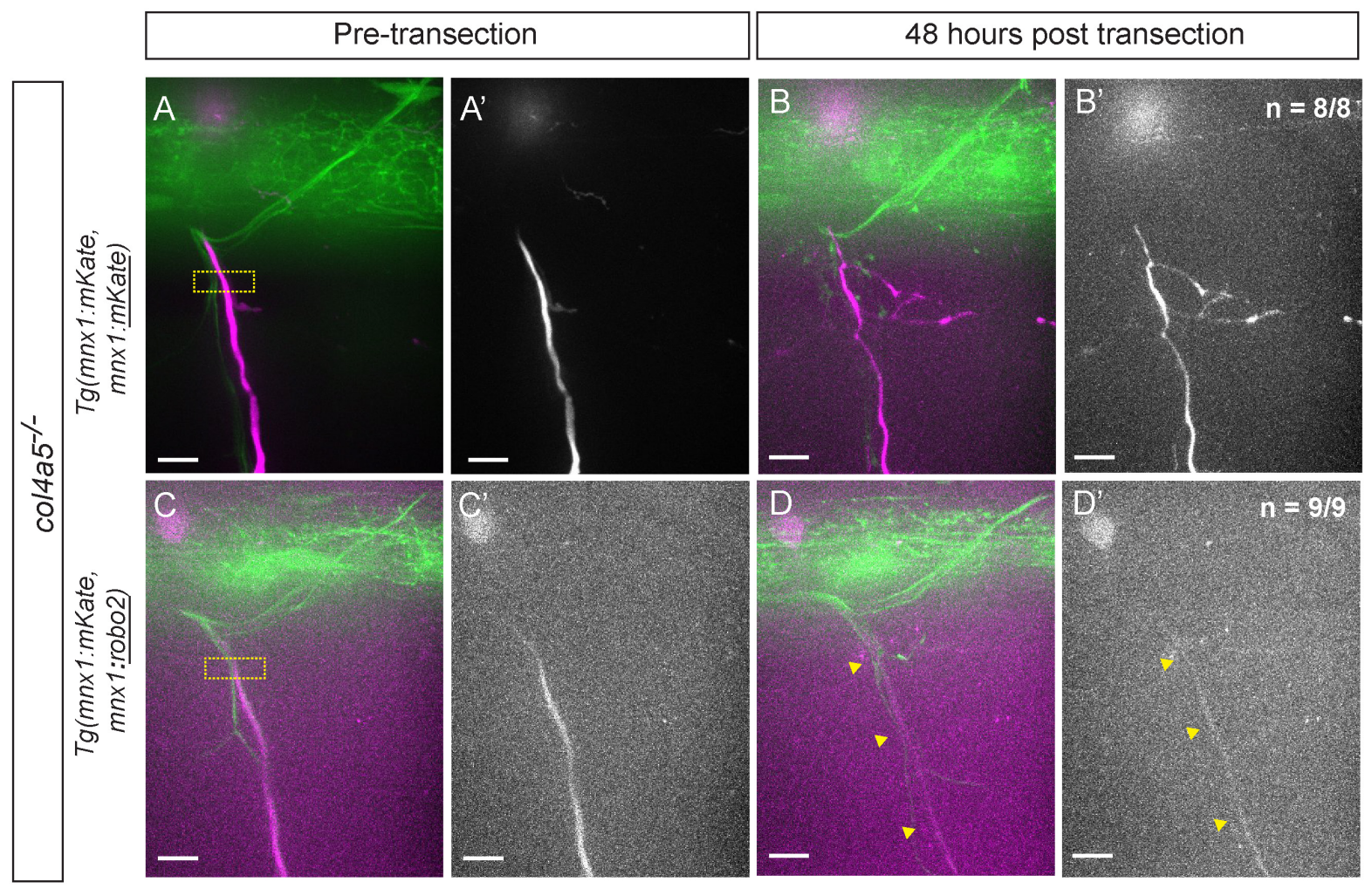

Fig. 5. col4a5 is required for the role of robo2 in branch-selective axon regeneration. Representative images of $T g(h b 9: G F P)$ (green) nerves in col4a5 $/-$ larvae with fascicles expressing transient $\operatorname{Tg}(m n x 1: m K a t e, m n x 1: m K a t e)$ (A-B) or $\operatorname{Tg}(m n x 1: m K a t e, ~ m n x 1: r o b o 2)$ (C-D) (magenta) in small subsets of ventrally projecting motor axons. Merged GFP and mKate images shown at $5 \mathrm{dpf}(\mathrm{A}, \mathrm{C})$ and $48 \mathrm{hpt}(\mathrm{B}, \mathrm{D})$. mKate channel is shown alone at $5 \mathrm{dpf}\left(\mathbf{A}^{\prime}, \mathbf{C}^{\prime}\right)$ and $48 \mathrm{hpt}$ (B', D'). In larvae expressing $T g(m n x 1: m K a t e, m n x 1: m K a t e), n=8 / 8$ nerves had only ventral regrowth of mKate ${ }^{+}$fascicles, like in the example shown. In larvae expressing $T g(m n x 1: m K a t e, ~ m n x 1: r o b o 2)$, $n=9 / 9$ nerves had only ventral regrowth of $\mathrm{mKate}^{+}$fascicles, like in the example shown. Proportions of ventral regrowth were compared between conditions using one-tailed Fischer's exact test $(p=0.999)$. Images were processed as described in Materials and Methods. Dashed yellow boxes, transection site. Scale bars, 10 um.

and explore the nerve branch-point before turning dorsally during regeneration (Fig. 3). Combined, this provides compelling evidence that rather than providing a permissive substrate and environment, spatially restricted col4a5 expression is critical to instruct regenerating dorsal nerve axons at the nerve branch-point.

Our results also reveal a previously uncharacterized role for role Slit-Robo signaling in target selective regeneration (Fig 5). Loss of function mutations in two Slit-Robo signaling components, extl3 and robo2, result in the same target selective regeneration defects (Fig. 2). Conversely, we find that transgenic expression of robo2 in ventral nerve axons, which are unaffected by the loss of Slit-Robo signaling, is sufficient to redirect these axons onto a dorsal nerve branch specific route (Fig. 4), and that this process requires Col4a5 function (Fig. 5). Given the incomplete penetrance of the robo 2 mutant phenotype in target selective regeneration, we cannot exclude the possibility that one or multiple of the additional three zebrafish Robo receptors ${ }^{48,49}$, play a role in this process and hence partially compensate for the loss of robo2. Future experiments, including generating single and double mutant combinations for the other three Robo receptors as well for each of the four known Slit ligands ${ }^{50}$ will provide a comprehensive view on role of Slit-Robo signaling during target selective regeneration and inform the contribution of individual Robo receptors in this process. Nonetheless, our results provide strong support for a mechanism in which glial-derived col4a5 expression restricted to the branch-point promotes dorsal turning of regenerating axons possibly via Col4a5-bound Slit1a. Moreover, the transient expression of col4a5 and slit la, which only lasts a few hours and coincides with the time period when regenerating axons navigate the branch choice point is remarkable, underscoring the high degree and functional importance of spatially and temporally signaling between regenerating axons and Schwann cells to achieve target selective regeneration. While our results identify a previously uncharacterized molecular mechanism to promote peripheral nerve regeneration, they also draw attention to the need to incorporate spatially and temporally restricted deliveries of guidance information in therapeutic strategies aimed at enhancing target selective regeneration. 
during regeneration

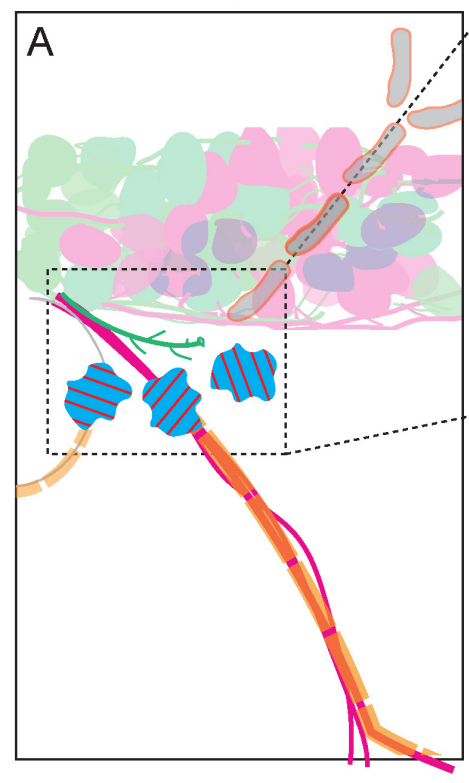

wildtype sibling

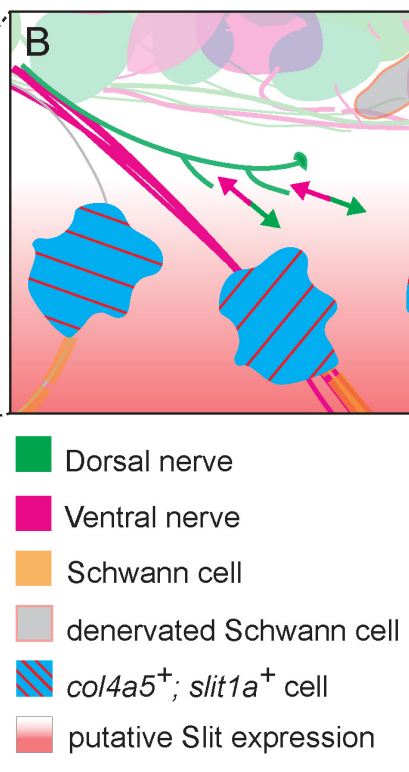

Fig. 6. Model for role of col4a5-robo2 pathway in target-selective axon regeneration. During regeneration, (A) a subset of specialized Schwann cells at the nerve branch-point express col4a5 (blue with red stripes), which scaffolds the repulsive axon guidance cue Slit (red gradient) in the local ECM. (B) In response to Slit at the nerve branch-point, regenerating wildtype axons expressing the Slit-receptor robo2 extend for short distances along ventral and ventro-lateral paths, as they navigate the nerve branch choice-point. These short erroneous extensions (green arrows) are balanced by retraction events (red arrows), which results in their eventual retraction and correction. (C) in robo2 $2^{-/-}$larvae, regenerating axons are not responsive to Slit at the nerve branch-point and thus extend more frequently along ventral and ventro-lateral paths. These frequent erroneous extensions (large green arrows) are not balanced by retraction events (red arrows), which occur with similar frequency as retractions in wildtype siblings. Thus, in robo2 ${ }^{-/-}$larvae, branch-point errors extend for longer distances and are less likely to be corrected.

\section{Materials and Methods}

Fish Lines and Maintenance. All fish lines were maintained in Tügbingen or Tupfel longfin (TLF) backgrounds and maintained as previously described ${ }^{51}$. We used the following mutant alleles, which were genotyped as previously described: robo2-ti272z $z^{52}$, extl3-tm70g ${ }^{27}$, col $4 a 5$ $s 510^{53}$.The $T g(\operatorname{sox} 10:$ colta5-Myc) line was generated as previously described ${ }^{12}$ and genotyped by amplifying the Myc transgene using the following primers: 5' GACTACAAGGATGACGATGACAAG 3' (forward) and 5' TTCTCCCATAGTCACGCTAGC 3' (reverse). For in vivo imaging, the following transgenic lines were used: $T g(m n x l: G F P)^{m l 329}$ to visualize motor axons in both dorsal and ventral nerve branches, $\operatorname{Tg}(i s l 1: G F P)^{r w 0} 54$ to visualize dorsal nerve branch axons alone. Zebrafish veterinary care was performed under the supervision of the University Laboratory Animal Resources (ULAR) at the University of Pennsylvania. All zebrafish work was performed in accordance with protocols approved by the University of Pennsylvania Institutional Animal Care and Use (IACUC).

Nerve Transection. Dorsal and ventral nerves were transected using a nitrogen-pulsed dye $(440 \mathrm{~nm})$ laser as previously described ${ }^{20}$. Briefly, one of the two nerve branches (dorsal for Figures 1, 2 and 3 or ventral for Figures 4 and 5 ) were transected $\sim 5 \mathrm{um}$ from the nerve branch-point (1015 um from the MEP), leaving the other nerve branch intact and a $\sim 5$ um gap between proximal and distal stumps of the transected nerve branch.

In extl $3^{-/-}$larvae, dorsal nerves reach dorsal muscle targets, but a small subset grow along an aberrantly lateral trajectory. This phenotype is variably penetrant, affecting 0$50 \%$ of nerves per larvae and $<20 \%$ of all nerves across the mutant population ( $\mathrm{n}>50$ larvae; PLM, unpublished observations). Thus, for dorsal nerve transections in extl3 $3^{-/-}$larvae, we carefully selected phenotypically normal nerves for transection.

Quantification of Axon Regeneration. Dorsal axon guidance pre- and post-transection was quantified using modified Sholl analysis, as previously described ${ }^{12}$, with the exception that line thickness in Sholl diagrams here do not correlate to fascicle thickness. To calculate the regeneration error rate for each nerve, we divided the number of fascicles that regenerated outside the dorsal ROI ("errors") by the total number of fascicles that regenerated by $48 \mathrm{hpt}$. To determine the angle of ventral nerve extension, we measured the angle between two consistent points along the trunk of the nerve (50um and 100um from the MEP). The extent of ventral nerve regeneration was scored by counting the number of fascicles which regenerated at least 50um from the MEP by $48 \mathrm{hpt}$.

Immunohistochemistry and whole-mount fluorescent in situ hybridization. To visualize robo 2 expression after nerve transection, dorsal nerves were transected in $5 \mathrm{dpf}$ Tg(isll:GFP) larvae. At 0-10 hpt, larvae were fixed in $4 \%$ 
PFA in PBS with $0.1 \%$ Tween- 20 overnight at $4 \circ \mathrm{C}$. The antisense robo 2 probe was synthesized from pBlueScriptrobo2 linearized with EcoRI using T3 RNA polymerase (Promega); the sense robo2 probe was synthesized from pBlueScript-robo2 linearized with XhoI using T7 RNA polymerase (Promega). Probes were hydrolyzed in $0.6 \mathrm{M}$ sodium carbonate and $0.4 \mathrm{M}$ sodium bicarbonate at $60^{\circ} \mathrm{C}$ for $11 \mathrm{~min}$ to yield 300-500bp fragments. Whole mount in situ hybridization was performed as previously described ${ }^{55}$ with the following modifications: $5 \mathrm{dpf}$ larvae were permeabilized by digesting with Proteinase K (10ug/ml, Promega) for 2 hours; endogenous peroxidases were quenched by incubating in $0.3 \% \mathrm{H}_{2} \mathrm{O}_{2}$ for 30 minutes before adding anti-digoxigenin antibody; for blocking and antibody incubation, we used $2 \%$ Blocking Reagent (Roche) in PBS with $0.1 \%$ Tween20; probes were detected using sheep anti-digoxigenin POD Fab fragments (Roche) and developed for 2 minutes using Tyramide Signal Amplification (TSA Plus kit, PerkinElmer). We stained for emphTg(isl1:GFP) using chicken anti-GFP (1:500, Aves Labs) detected by donkey anti-chick Alexa Fluor 488 (1:500, Jackson ImmunoResearch Laboratories, Inc.). Anti-digoxigenin POD and anti-GFP primary antibodies were incubated concurrently; secondary antibody was incubated at $4{ }^{\circ} \mathrm{C}$ overnight after TSA.

Nerves were imaged in 1 um sections using a $63 \mathrm{X}$ water immersion lens on a Zeiss LSM 880 laser scanning confocal microscope and Zeiss Zen software or using a $40 \mathrm{X}$ water immersion lens on an Olympus Spinning disk confocal microscope and 3i Slidebook Software. Overlap between emphTg(isl1:GFP) and emphrobo2 probe was quantified from 40X images in Fiji in the following way: motor pools of transected nerves were isolated by cropping optical sections in 3D; motor pools were then compressed into MIPs; GFP and robo2 probe signals were separated and converted into binary masks using max entropy and moments methods, respectively; percent particle overlap between the two masks was calculated using the GDSC colocalization plugin; percent particle overlap was then normalized to the number of cell bodies manually counted in each motor pool.

Plasmid construction. The mnx1:mKate, mnx1:robo2 plasmid was constructed using Gateway cloning ${ }^{56}$ using pME:robo $2^{57}$ and pI-SceI mnx1:mKate, mnx1:DEST, which encodes two mnx1 promoters in tandem ${ }^{58}$, to insert the robo 2 coding sequence behind the second $\mathrm{mnx} 1$ promoter. To construct plasmid for synthesis of robo2 in situ probe (pBlueScript-robo2), the full length robo2 coding sequence was amplified from the mnx1:mKate, mnx1:robo2 plasmid using the following primers 5' AGTCAGCTCGAGAACGTGTTCTGGGGT-TGAGA 3' (forward, includes XhoI restriction site) and 5'GCTAACGAATTCTGGGTATGAGGCATTTCCAGAAC 3' (reverse, includes EcoRI restriction site). XhoI and EcoRI restriction sites were used to clone this product into pBluescript II KS+.

Sparse axonal labeling. We used mnx1:mKate, $\mathrm{mnx} 1$ :robo2 and $\mathrm{mnx} 1 \mathrm{mKate}, \mathrm{mnx} 1 \mathrm{mKate}$ to label small numbers of ventral motor neurons by injecting 50-100 pg of plasmid DNA into one-cell stage embryos with Isce-I, as previously described ${ }^{59}$. We have previously validated that both $\mathrm{mnx} 1$ promoters in this construct are active and drive comparable levels of expression (ref. ${ }^{58}$ and unpublished observations). At $3 \mathrm{dpf}$, injected larvae with mKate expression were screened for ventral nerves with very few labeled axons using a $40 \mathrm{X}$ water immersion lens on a Olympus spinning disk confocal microscope using 3i Slidebook software. When examining developmental labeling frequency, nerves were scored as "dorsal" if any mKate ${ }^{+}$fascicles were present were present along the dorsal branch, regardless of whether $\mathrm{mKate}^{+}$fascicles were also present along the ventral branch.

It was technically challenging to sparsely label ventral axons in sufficient numbers for transection experiments without labeling multiple neurons per motor pool. We could almost always count multiple (2-6) mKate ${ }^{+}$cell bodies in motor pools corresponding to transected ventral nerves (data not shown). After ventral nerve transection, we often observed that emphTg(mnx1:mKate;mnx1:robo 2$)^{+}$fascicles regenerated along both the dorsal and the ventral branch (see Figure $5 \mathrm{D})$. We believe it is very likely that this reflects the regeneration of multiple labeled axons, rather than single bifurcating axons. Therefore, at $48 \mathrm{hpt}$, we scored $\mathrm{mKate}^{+}$fascicle regeneration as "ventral," if mKate ${ }^{+}$fascicles were observed only on the ventral nerve branch, and we scored mKate ${ }^{+}$fascicle regeneration as "dorsal" if we observed any mKate ${ }^{+}$ along the dorsal branch, regardless of whether $\mathrm{mKate}^{+}$fascicles were also present along the ventral branch.

Live Imaging. Larvae were anesthetized, mounted in agarose and imaged on a spinning disk confocal microscope as previously described ${ }^{20}$. We began our timelapse experiments 7-9 hpt and filmed regeneration for 12-15 hours. Due to variability in time when axons started regrowing (8-14 hpt), we quantified axon dynamics starting when the first regenerating fascicle reached the nerve branch-point and ending up to 10 hours later. We analyzed regenerating fascicles for a total of 5940 minutes in siblings ( $n=9$ nerves) and 5030 minutes in mutants ( $\mathrm{n}=10$ nerves).

Image Processing. For ventral nerves (Fig. S2) and fixed samples (Fig. S1), Z-stacks were compressed into maximum intensity projections (MIPs). Brightness and contrast were automatically optimized based upon the image histogram in Fiji ImageJ (NIH). The dorsal nerve branch wraps around the spinal cord, closely apposed to motor neuron cell bodies, which are labeled brightly by our transgenic lines. To visualize the dorsal nerve independently of neuron cell bodies (Figs. 1, 2, 3, 4, 5), we used Fiji to create multiple MIPs from the same Z-stack, including only optical sections that contained the dorsal nerve without neuron cell bodies in each $\mathrm{XY}$ position. These MIPs were adjusted to equivalent brightness and contrast and then stitched together using the Pairwise Stitching plugin /citePreibisch2009.

Statistical Analyses. Continuous data (Fig. 3) were analyzed using one- or two-tailed t-tests, as indicated in figure 
legends. Categorical data (Figs. 1-5) were analyzed in contingency tables using one- or two-tailed Fisher's exact tests for proportionality, as indicated in figure legends. Count data (Fig. 3) were analyzed using two-tailed Mann Whitney tests.

\section{References}

1. Kai Liu, Andrea Tedeschi, Kevin Kyungsuk Park, and Zhigang He. Neuronal intrinsic mechanisms of axon regeneration. Annual Review of Neuroscience, 34:131-152, 2011. ISSN 0147006X. doi: 10.1146/annurev-neuro-061010-113723.

2. Mark A. Anderson, Timothy M. O'Shea, Joshua E. Burda, Yan Ao, Sabry L. Barlatey, Alexander M. Bernstein, Jae H. Kim, Nicholas D. James, Alexandra Rogers, Brian Kato, Alexander L. Wollenberg, Riki Kawaguchi, Giovanni Coppola, Chen Wang, Timothy J. Deming, Zhigang He, Gregoire Courtine, and Michael V. Sofroniew. Required growth facilitators propel axon regeneration across complete spinal cord injury. Nature, 561(7723):396-400, 2018. ISSN 14764687. doi: 10.1038/s41586-018-0467-6.

3. Cédric G. Geoffroy, Ariana O. Lorenzana, Jeffrey P. Kwan, Kyle Lin, Omeed Ghassemi, Andrew Ma, Nuo Xu, Daniel Creger, Kai Liu, Zhigang He, and Binhai Zheng. Effects of PTEN and Nogo codeletion on corticospinal axon sprouting and regeneration in mice. Journal of Neuroscience, 35(16):6413-6428, 2015. ISSN 15292401. doi: 10.1523/JNEUROSCI. 4013-14.2015.

4. Fang Sun, Kevin K. Park, Stephane Belin, Dongqing Wang, Tao Lu, Gang Chen, Kang Zhang, Cecil Yeung, Guoping Feng, Bruce A. Yankner, and Zhigang He. Sustained axon regeneration induced by co-deletion of PTEN and SOCS3. Nature, 480(7377):372-375, 2011. ISSN 00280836. doi: 10.1038/nature 10594

5. Andrea Tedeschi, Sebastian Dupraz, Michele Curcio, Claudia J. Laskowski, Barbara Schaffran, Kevin C. Flynn, Telma E. Santos, Sina Stern, Brett J. Hilton, Molly J.E. Larson, Christine B. Gurniak, Walter Witke, and Frank Bradke. ADF/Cofilin-Mediated Actin Turnover Promotes Axon Regeneration in the Adult CNS. Neuron, 103(6):1073-1085.e6, 2019. ISSN 10974199. doi: 10.1016/j.neuron.2019.07.007.

6. Sydney Sunderland. Nerve injuries and their repair: a critical appraisal. Churchill Livingstone, New York, 1991.

7. Roger D. Madison, G. A. Robinson, and S. R. Chadaram. The specificity of motor neurone regeneration (preferential reinnervation). Acta Physiologica, 189(2):201-206, 2007. ISSN 17481708. doi: 10.1111/j.1748-1716.2006.01657.x.

8. Arthur W English. Enhancing axon regeneration in peripheral nerves also increases functionally inappropriate reinnervation of targets. Journal of Comparative Neurology, 490(4): 427-441, 2005. ISSN 1096-9861. doi: 10.1002/cne.20678.

9. Tessa Gordon and Arthur W. English. Strategies to promote peripheral nerve regeneration: Electrical stimulation and/or exercise. European Journal of Neuroscience, 43(3):336-350, 2016. ISSN 14609568. doi: 10.1111/ejn.13005.

10. Roman J. Giger, Edmund R. Hollis, and Mark H. Tuszynski. Guidance molecules in axon regeneration. Cold Spring Harbor perspectives in biology, 2(7):1-22, 2010. ISSN 19430264. doi: 10.1101/cshperspect.a001867.

11. Cynthia Lance-Jones and Lynn Landmesser. Pathway selection by chick lumbosacral motoneurons during normal development. Proceedings of the Royal Society of London - Biological Sciences, 214(1194):1-18, 1981. ISSN 09628452. doi: 10.1098/rspb.1981.0079.

12. Jesse Isaacman-Beck, Valerie Schneider, Clara Franzini-Armstrong, and Michael Granato. The Ih3 Glycosyltransferase Directs Target-Selective Peripheral Nerve Regeneration. Neuron, 88(4):691-703, 2015. ISSN 10974199. doi: 10.1016/j.neuron.2015.10.004.

13. Richard Redett, Rajesh Jari, Thomas Crawford, You Gang Chen, Charles Rohde, and Thomas M. Brushart. Peripheral pathways regulate motoneuron collateral dynamics. Journal of Neuroscience, 25(41):9406-9412, 2005. ISSN 02706474. doi: 10.1523/JNEUROSCI. 3105-05.2005.

14. Richard F. Mark. Fin movement after regeneration of neuromuscular connections: An investigation of myotypic specificity. Experimental Neurology, 12(3):292-302, 1965. ISSN 10902430. doi: 10.1016/0014-4886(65)90073-7.

15. Michael J. Politis. Specificity in mammalian peripheral nerve regeneration at the level of the nerve trunk. Brain Research, 328(2):271-276, 1985. ISSN 00068993. doi: 10.1016/ 0006-8993(85)91038-8.

16. David Choi and Geoffrey Raisman. After facial nerve damage, regenerating axons become aberrant throughout the length of the nerve and not only at the site of the lesion: An experimental study. British Journal of Neurosurgery, 18(1):45-48, 2004. ISSN 02688697. doi: 10.1080/02688690410001660454.

17. Godard C.W. De Ruiter, Robert J. Spinner, Joost Verhaagen, and Martijn J.A. Maless. Misdirection and guidance of regenerating axons after experimental nerve injury and repair: A review. Journal of Neurosurgery, 120(2):493-501, 2014. ISSN 00223085. doi: 10.3171/2013.8.JNS122300.

18. Thomas M. Brushart and M. Marsel Mesulam. Alteration in connections between muscle and anterior horn motoneurons after peripheral nerve repair. Science, 208(4444):603-605, 1980. ISSN 00368075. doi: $10.1126 /$ science. 7367884 .

19. Jun Kimura, Robert L. Rodnitzky, and Shige Hisa Okawara. Electrophysiologic analysis of aberrant regeneration after facial nerve paralysis. Neurology, 25(10):989-93, 1975. ISSN 1526632X. doi: 10.1212/wnl.25.10.989.

20. Allison F. Rosenberg, Marc A. Wolman, Clara Franzini-Armstrong, and Michael Granato. In Vivo Nerve-Macrophage Interactions Following Peripheral Nerve Injury. Journal of Neuroscience, 32(11):3898-3909, 2012. ISSN 0270-6474. doi: 10.1523/JNEUROSCI.5225-11. 2012.

21. Paul Z. Myers, Judith S. Eisen, and Monte Westerfield. Development and axonal outgrowth of identified motoneurons in the zebrafish. Journal of Neuroscience, 6(8):2278-2289, 1986. ISSN 02706474. doi: 10.1523/jneurosci.06-08-02278.1986.

22. W. van Raamsdonk, W. Mos, M. J. Smit-Onel, W. J. van der Laarse, and R. Fehres. The development of the spinal motor column in relation to the myotomal muscle fibers in the zebrafish (Brachydanio rerio) - I. Posthatching development. Anatomy and Embryology, 167(1):125-139, 1983. ISSN 03402061. doi: 10.1007/BF00304606.

23. Fabian N. Svara, Jörgen Kornfeld, Winfried Denk, and Johann H. Bollmann. Volume EM Reconstruction of Spinal Cord Reveals Wiring Specificity in Speed-Related Motor Circuits. Cell Reports, 23(10):2942-2954, 2018. ISSN 22111247. doi: 10.1016/j.celrep.2018.05.023.

24. Monte Westerfield, J. V. McMurray, and J. S. Eisen. Identified motoneurons and their innervation of axial muscles in the zebrafish. Journal of Neuroscience, 6(8):2267-2277, 1986. ISSN 02706474. doi: 10.1523/jneurosci.06-08-02267.1986.

25. Tong Xiao, Wendy Staub, Estuardo Robles, Nathan J. Gosse, Gregory J. Cole, and Herwig Baier. Assembly of lamina-specific neuronal connections by slit bound to type IV collagen. Cell, 146(1):164-176, 2011. ISSN 00928674. doi: 10.1016/j.cell.2011.06.016.

26. Heike Blockus and Alain Chédotal. Slit-robo signaling. Development (Cambridge), 143(17): 3037-3044, 2016. ISSN 14779129. doi: 10.1242/dev.132829.

27. Jeong Soo Lee, Sophia Von Der Hardt, Melissa A. Rusch, Sally E. Stringer, Heather L. Stickney, William S. Talbot, Robert Geisler, Christiane Nüsslein-Volhard, Scott B. Selleck, Chi Bin Chien, and Henry Roehl. Axon sorting in the optic tract requires HSPG synthesis by ext2 (dackel) and extl3 (boxer). Neuron, 44(6):947-960, 2004. ISSN 08966273. doi: 10.1016/j.neuron.2004.11.029.

28. Sadaf Ahmahni Hussain, Michael Piper, Noémi Fukuhara, Laure Strochlic, Gian Cho, Jason A. Howitt, Yassir Ahmed, Andrew K. Powell, Jeremy E. Turnbull, Christine E. Holt, and Erhard Hohenester. A molecular mechanism for the heparan sulfate dependence of slit-robo signaling. Journal of Biological Chemistry, 281(51):39693-39698, 2006. ISSN 00219258. doi: 10.1074/jbc.M609384200.

29. Heather Flanagan-Steet, Michael A. Fox, Dirk Meyer, and Joshua R. Sanes. Neuromuscular synapses can form in vivo by incorporation of initially aneural postsynaptic specializations. Development, 132(20):4471-4481, 2005. ISSN 09501991. doi: 10.1242/dev.02044.

30. Thomas M. Brushart. Motor axons preferentially reinnervate motor pathways. Journal of Neuroscience, 13(6):2730-2738, 1993. doi: 10.1523/jneurosci.13-06-02730.1993.

31. Christian Krarup, Simon J. Archibald, and Roger D. Madison. Factors that influence peripheral nerve regeneration: An electrophysiological study of the monkey median nerve. Annals of Neurology, 51(1):69-81, 2002. ISSN 03645134. doi: 10.1002/ana.10054.

32. G. Lundborg, Lars B. Dahlin, Nils Danielsen, and Ann K. Nachemson. Tissue specificity in nerve regeneration. Scandinavian Journal of Plastic and Reconstructive Surgery and Hand Surgery, 1986. ISSN 02844311. doi: 10.3109/02844318609004486.

33. Quyen T. Nguyen, Joshua R. Sanes, and Jeff W. Lichtman. Pre-existing pathways promote precise projection patterns. Nature Neuroscience, 5(9):861-867, 2002. ISSN 10976256. doi: $10.1038 / \mathrm{nn} 905$.

34. Roger W. Sperry and H. L. Arora. Selectivity in regeneration of the oculomotor nerve in the cichlid fish, Astronotus ocellatus. Journal of Embryology and Experimental Morphology, 14 (3):307-317, 1965. ISSN 00220752.

35. Seita Hagino, Ken Iseki, Tetsuji Mori, Yuxiang Zhang, Tsuyoshi Hikake, Sachihiko Yokoya, Mayumi Takeuchi, Hiromi Hasimoto, Shinichi Kikuchi, and Akio Wanaka. Slit and glypican-1 mRNAs are coexpressed in the reactive astrocytes of the injured adult brain. Glia, 42(2): 130-138, 2003. ISSN 08941491. doi: 10.1002/glia.10207.

36. Lizhen Chen, Zhiping Wang, Anindya Ghosh-Roy, Thomas Hubert, Dong Yan, Sean O'Rourke, Bruce Bowerman, Zilu Wu, Yishi Jin, and Andrew D. Chisholm. Axon Regeneration Pathways Identified by Systematic Genetic Screening in C. elegans. Neuron, 71(6): 1043-1057, 2011. ISSN 08966273. doi: 10.1016/j.neuron.2011.07.009.

37. Madeel Abdullah, Andres O'Daly, Alka Vyas, Charles Rohde, and Thomas M. Brushart. Adult motor axons preferentially reinnervate predegenerated muscle nerve. Experimental Neurology, 249:1-7, 2013. ISSN 00144886. doi: 10.1016/j.expneurol.2013.07.019.

38. R. D. Madison, M. V. Sofroniew, and G. A. Robinson. Schwann cell influence on moto neuron regeneration accuracy. Neuroscience, 163(1):213-221, 2009. ISSN 03064522. doi: 10.1016/j.neuroscience.2009.05.073.

39. Thomas M. Brushart, M. Aspalter, J. W. Griffin, R. Redett, H. Hameed, C. Zhou, M. Wright, A. Vyas, and A. Höke. Schwann cell phenotype is regulated by axon modality and centralperipheral location, and persists in vitro. Experimental Neurology, 247:272-281, 2013. ISSN 00144886. doi: 10.1016/j.expneurol.2013.05.007.

40. Ahmet Höke, Richard Redett, H. Hameed, R. Jari, C. Zhou, Z. B. Li, J. W. Griffin, and T. M. Brushart. Schwann cells express motor and sensory phenotypes that regulate axon regeneration. Journal of Neuroscience, 26(38):9646-9655, 2006. ISSN 02706474. doi: 10.1523/JNEUROSCI.1620-06.2006.

41. Nithya J. Jesuraj, Peter K. Nguyen, Matthew D. Wood, Amy M. Moore, Gregory H. Borschel, Susan E. Mackinnon, and Shelly E. Sakiyama-Elbert. Differential gene expression in motor and sensory Schwann cells in the rat femoral nerve. Journal of Neuroscience Research, 90 (1):96-104, 2012. ISSN 03604012. doi: 10.1002/jnr.22752.

42. Matthew D. Wood and Susan E. Mackinnon. Pathways regulating modality-specific axonal regeneration in peripheral nerve. Experimental Neurology, 265:171-175, 2015. ISSN 10902430. doi: 10.1016/j.expneurol.2015.02.001.

43. Colin K. Franz, Urs Rutishauser, and Victor F. Rafuse. Polysialylated neural cell adhesion molecule is necessary for selective targeting of regenerating motor neurons. Journal of Neuroscience, 25(8):2081-2091, 2005. ISSN 02706474. doi: 10.1523/JNEUROSCI. 4880-04.2005.

44. Rudolf Martini, M. Schachner, and T. M. Brushart. The L2/HNK-1 carbohydrate is preferentially expressed by previously motor axon-associated Schwann cells in reinnervated peripheral nerves. Journal of Neuroscience, 14(11):7180-7191, 1994. ISSN 02706474. doi: 10.1523/jneurosci.14-11-07180.1994.

45. N. Ghalib, L. Houšvt'ava, P. Haninec, and P. Dubový. Morphometric analysis of early regeneration of motor axons through motor and cutaneous nerve grafts. Annals of Anatomy, 183 (4):363-368, 2001. ISSN 09409602. doi: 10.1016/S0940-9602(01)80183-7.

46. K R Jessen and R Mirsky. The repair Schwann cell and its function in regenerating. 13(July 2015):3521-3531, 2016. doi: 10.1113/JP270874.

47. Kristjan R. Jessen and Rhona Mirsky. The success and failure of the schwann cell response to nerve injury. Frontiers in Cellular Neuroscience, 13(33):1-14, 2019. ISSN 16625102. doi: 10.3389/fncel.2019.00033.

48. Victoria M. Bedell, Sang Yeob Yeo, Won Park Kye, Jeffrey Chung, Pankaj Seth, Venkate- 
bioRxiv preprint doi: https://doi.org/10.1101/2020.12.07.406942; this version posted December 7, 2020. The copyright holder for this preprint (which was not certified by peer review) is the author/funder. All rights reserved. No reuse allowed without permission.

sha Shivalingappa, Jinhua Zhao, Tomoko Obara, Vikas P. Sukhatme, lain A. Drummond, Dean Y. Li, and Ramani Ramchandran. roundabout4 is essential for angiogenesis in vivo. Proceedings of the National Academy of Sciences of the United States of America, 102(18): 6373-8, 2005. ISSN 00278424. doi: 10.1073/pnas.0408318102.

49. Jeong Soo Lee, Russell Ray, and Chi Bin Chien. Cloning and expression of three zebrafish roundabout homologs suggest roles in axon guidance and cell migration. Developmental Dynamics, 221(2):216-230, 2001. ISSN 10588388. doi: 10.1002/dvdy.1136.

50. Lara D. Hutson, Michael J. Jurynec, Sang Yeob Yeo, Hitoshi Okamoto, and Chi B. Chien. Two Divergent slit1 Genes in Zebrafish. Developmental Dynamics, 228(3):358-369, 2003. ISSN 10588388. doi: 10.1002/dvdy. 10386 .

51. Mary C. Mullins, Matthias Hammerschmidt, Pascal Haffter, and Christiane NüssleinVolhard. Large-scale mutagenesis in the zebrafish: in search of genes controlling development in a vertebrate. Current Biology, 4(3):189-202, 1994. ISSN 09609822. doi: 10.1016/S0960-9822(00)00048-8.

52. Cornelia Fricke, Jeong-Soo Lee, Silke Geiger-Rudolph, Friedrich Bonhoeffer, and ChiBin Chien. Astray, a Zebrafish Roundabout Homolog Required for Retinal Axon Guidance. Science (New York, N.Y.), 292(5516):507-510, 2001. ISSN 0036-8075. doi: $10.1126 /$ science. 1059496 .

53. Tong Xiao and Herwig Baier. Lamina-specific axonal projections in the zebrafish tectum require the type IV collagen Dragnet. Nature Neuroscience, 10(12):1529-1537, 2007. ISSN 10976256. doi: 10.1038/nn2002.

54. Osamu Uemura, Yohei Okada, Hideki Ando, Mickael Guedj, Shin Ichi Higashijima, Takuya Shimazaki, Naoichi Chino, Hideyuki Okano, and Hitoshi Okamoto. Comparative functional genomics revealed conservation and diversification of three enhancers of the is 11 gene for motor and sensory neuron-specific expression. Developmental Biology, 278(2):587-606, 2005. ISSN 00121606. doi: 10.1016/j.ydbio.2004.11.031.

55. Christine Thisse and Bernard Thisse. High-resolution in situ hybridization to whole-mount zebrafish embryos. Nature Protocols, 3:59-69, 2008. ISSN 17542189. doi: 10.1038/nprot. 2007.514.

56. Kristen M. Kwan, Esther Fujimoto, Clemens Grabher, Benjamin D. Mangum, Melissa E. Hardy, Douglas S. Campbell, John M. Parant, H. Joseph Yost, John P. Kanki, and Chi Bin Chien. The Tol2kit: A multisite gateway-based construction Kit for Tol2 transposon transgenesis constructs. Developmental Dynamics, 236(11):3088-99, 2007. ISSN 10588388. doi: $10.1002 /$ dvdy. 21343 .

57. Douglas S. Campbell, Sydney a. Stringham, Adam Timm, Tong Xiao, Mei Yee Law, Herwig Baier, Michael L. Nonet, and Chi Bin Chien. Slit1a Inhibits Retinal Ganglion Cell Arborization and Synaptogenesis via Robo2-Dependent and -Independent Pathways. Neuron, 55(2) 231-245, 2007. ISSN 08966273. doi: 10.1016/j.neuron.2007.06.034.

58. Juliane Bremer and Michael Granato. Myosin phosphatase Fine-tunes Zebrafish Motoneuron Position during Axonogenesis. PLoS Genetics, 12(11):1-20, 2016. ISSN 15537404 doi: 10.1371/journal.pgen.1006440.

59. Gerald B. Downes, Julie A. Waterbury, and Michael Granato. Rapid in vivo labeling of identified zebrafish neurons. Genesis, 34(3):196-202, 2002. ISSN 1526954X. doi: 10. 1002 /gene. 10120 .

\section{AUTHOR CONTRIBUTIONS}

Patricia L. Murphy: Conceptualization, Methodology, Validation, Formal Analysis, Investigation, Data Curation, Writing - Original Draft Preparation, Writing - Review \& Editing, Visualization, Funding Acquisition

Jesse Isaacman-Beck: Conceptualization, Methodology, Investigation, Writing Review \& Editing

Michael Granato: Conceptualization, Resources, Writing - Review \& Editing, Supervision, Funding Acquisition

\section{ACKNOWLEDGEMENTS}

The authors would like to thank Daniel Morales, Leah Middleton, and Lauren Walker for technical assistance. We would also like to thank Dr. Andrea Stout of the Penn Cell and Developmental Biology microscopy core and the Penn sanger sequencing core for technical support. Finally, we would like to thank PLM's thesis committee members Dr. Wenqin Luo, Dr. Steven Scherer and Dr. Stephen DiNardo, as well as past and present members of the Granato lab, for helpful feedback on data and the manuscript. This article was typeset in Overleaf using the Henriques Lab BioRXiv template with minor modifications.

\section{COMPETING INTERESTS STATEMENT}

The authors declare that no competing interests exist.

\section{FUNDING}

This work was supported by 01 NS097914 awarded to MG, and T32HD083185 and

1F31NS103394 awarded to PLM. 

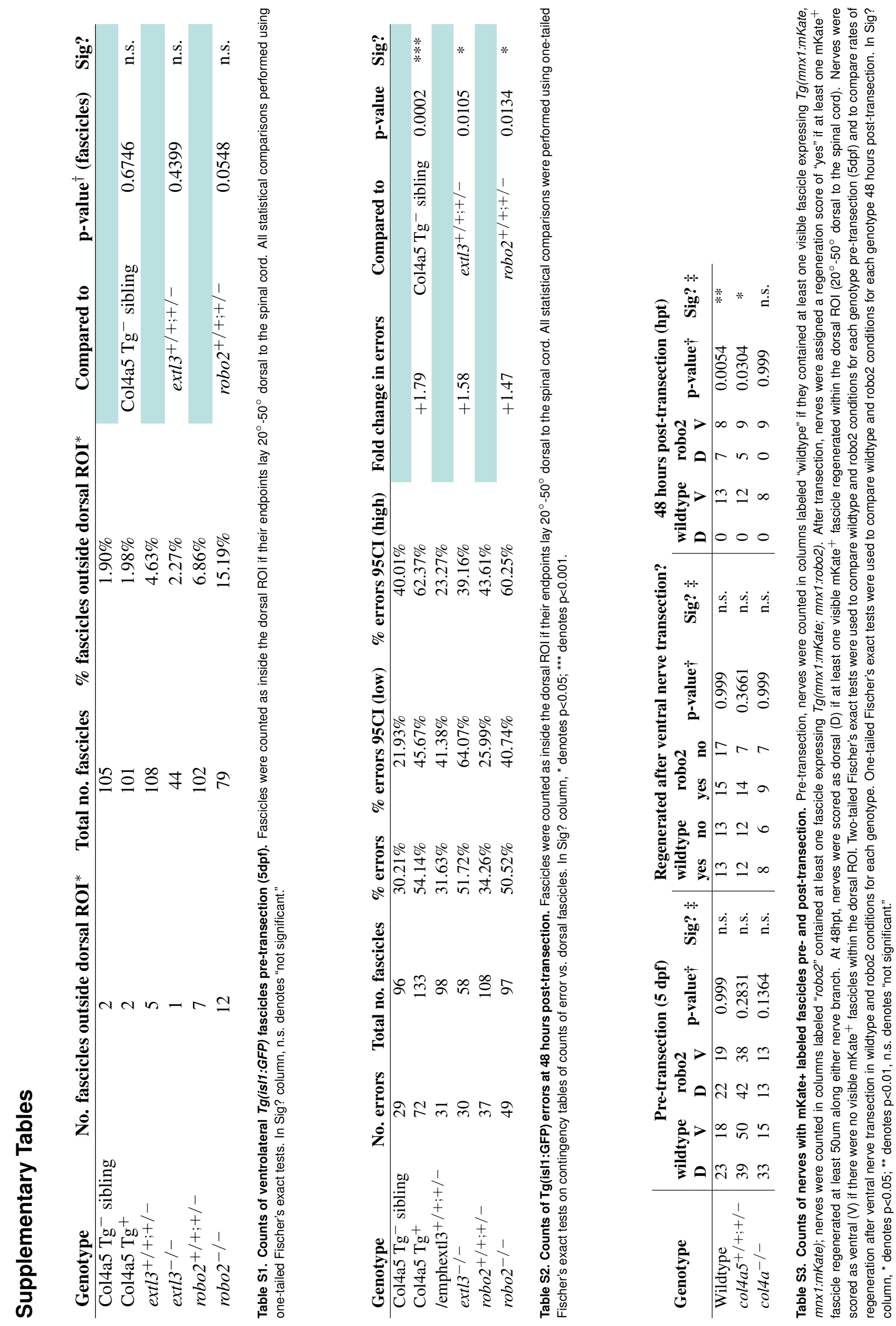
bioRxiv preprint doi: https://doi.org/10.1101/2020.12.07.406942; this version posted December 7, 2020. The copyright holder for this preprint (which was not certified by peer review) is the author/funder. All rights reserved. No reuse allowed without permission.

\section{Supplementary Figures}
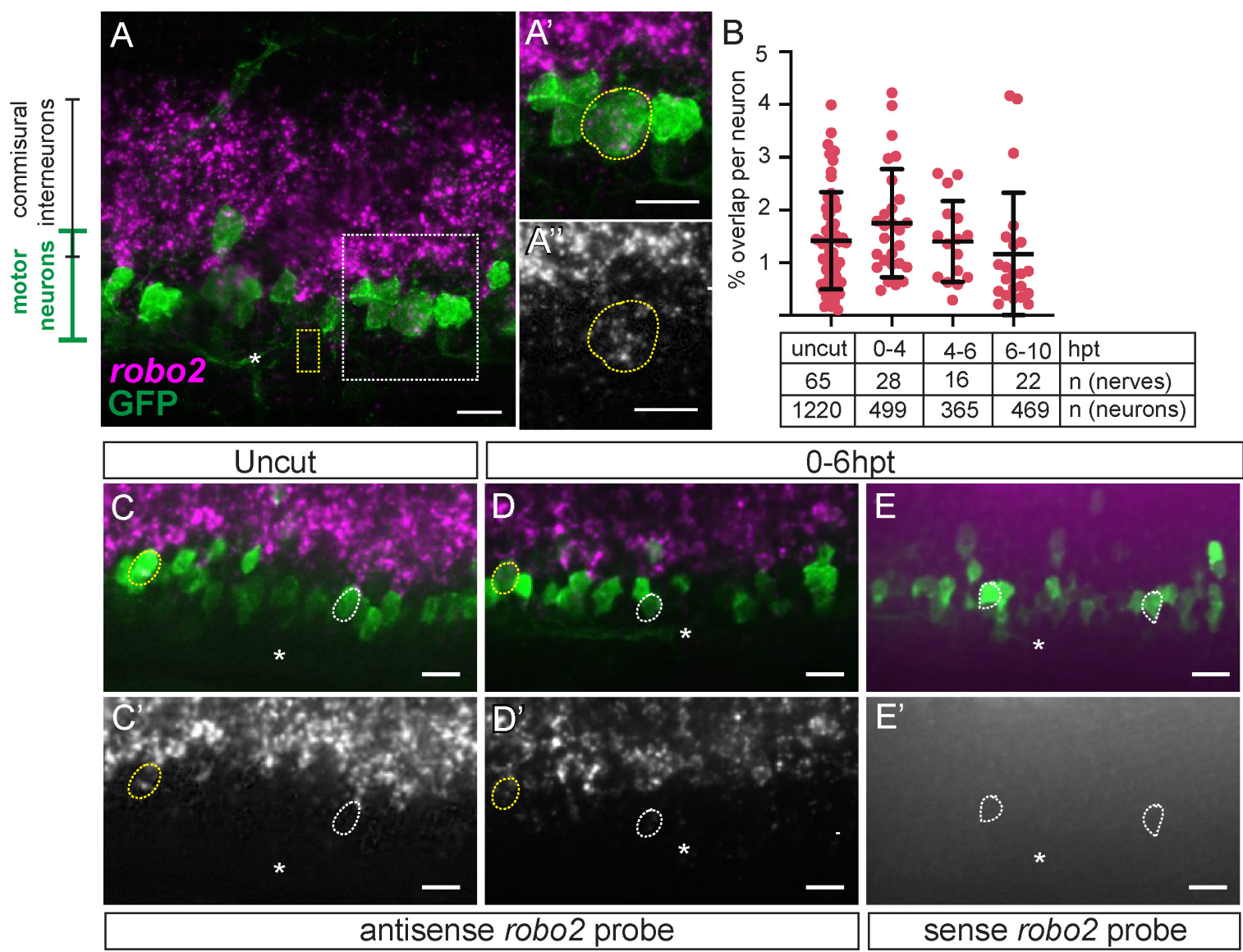

Fig. S1. Robo2 is expressed in is/1+ motor neurons before and after transection.(A) Representative image of $\operatorname{Tg}($ is/1:GFP) motor neurons in spinal cord 8-10 hpt stained with robo2 antisense ISH probe (magenta) and GFP antibody (green). Image shown is maximum Z-projection of 24 optical sections (10um), 63X. Dashed yellow box, transection site. Dashed white box, area enlarged 1.5X in (A') and (A") showing a single optical section (0.41um) with one $T$ g(is/1:GFP) motor neuron (green, outlined with yellow dashed line) expressing robo2 mRNA (magenta) merged (A') and alone (gray) (A"). (B) Quantification of colocalization of robo2 ISH probe with Tg(is/1:GFP) neurons at 0-10 hpt. Each dot represents the fraction of robo2 expression in a single motor pool that colocalized with GFP, normalized to the number of labeled motor neurons. Mean $\%$ overlap per neuron was compared across timepoints using one-way ANOVA $(p=0.2138)$. (C-E) Representative images of $T g($ is $/ 1$ :GFP) motor neurons stained with robo2 antisense ISH probe (C-D) or sense ISH probe control (E) (magenta) and GFP antibody (green) (merged). (C'-E') ISH probe of corresponding image alone (gray). Images shown are single optical sections (1um), 40X. of nerves untransected (C) and at 0-6 hpt (D-E). White asterisks, motor exit points (MEPs). Yellow dashed lines outline cell bodies with expression, white dashed lines outline cell bodies without expression. Scale bars 10um. 
bioRxiv preprint doi: https://doi.org/10.1101/2020.12.07.406942; this version posted December 7, 2020. The copyright holder for this preprint

(which was not certified by peer review) is the author/funder. All rights reserved. No reuse allowed without permission.
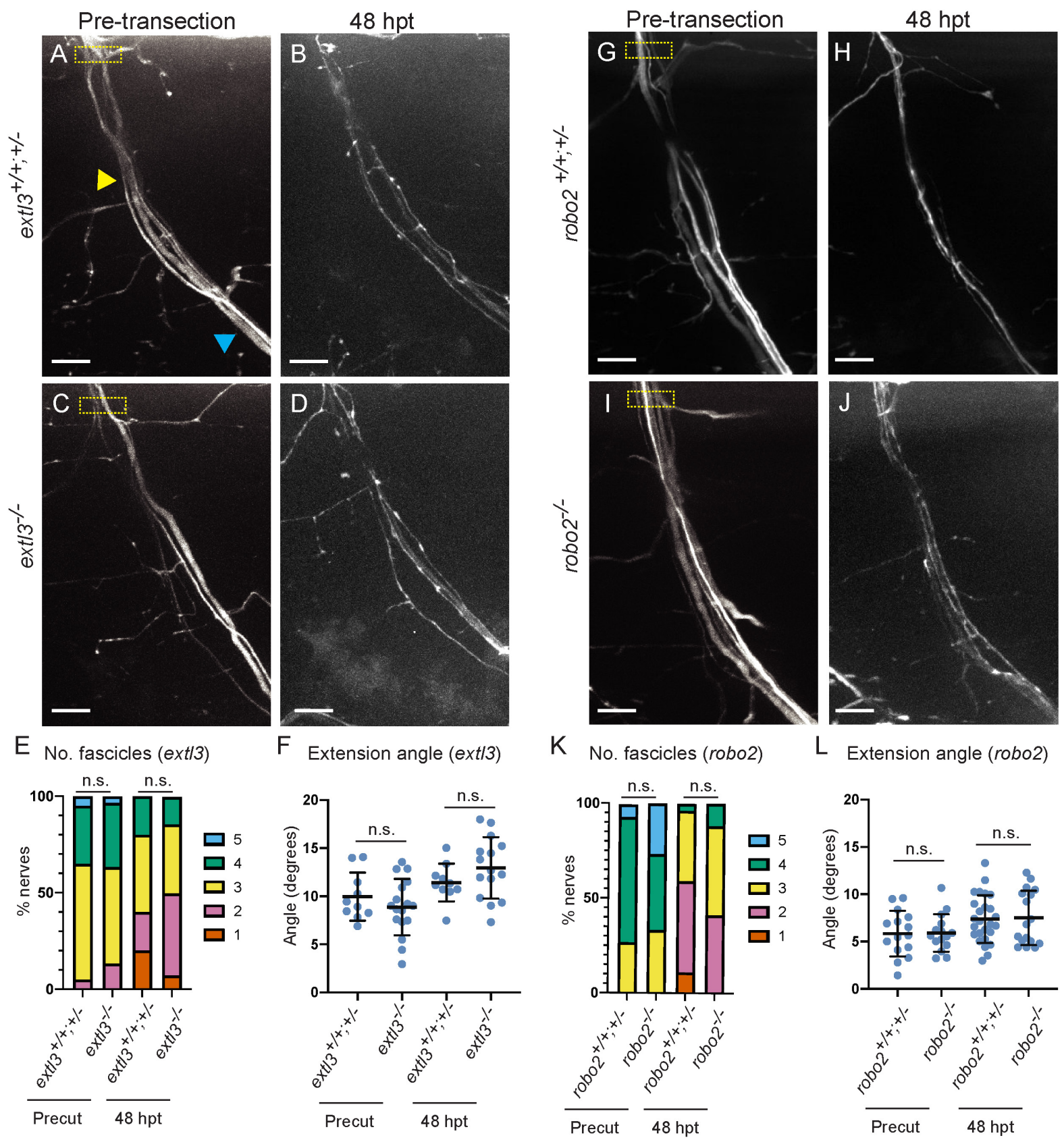

Fig. S2. Slit-Robo signaling is dispensable for ventral nerve branch development and regeneration. Top: Representative images of $T g(h b 9: G F P)$ ventral nerves at $5 \mathrm{dpf}$ and $48 \mathrm{hpt}$, respectively, in ext/3 siblings $(\mathbf{A}, \mathbf{B})$ ext/3 ${ }^{-/-}$(C, D), robo2 wildtype siblings $(\mathbf{G}, \mathbf{H})$, robo2 ${ }^{-/-}(\mathbf{I}, \mathbf{J})$. Motor exit point (MEP) is just dorsal to the top of imaging frame shown. Arrowheads in (A) mark 50um (yellow arrowhead) and 100um (blue arrowhead) from the MEP. Dashed yellow boxes denote transection site (10-15um ventral to MEP). Bottom: Quantification of ventral nerve fascicles at $5 \mathrm{dpf}$ (Precut) and $48 \mathrm{hpt}$ in $(\mathbf{E})$ extl3 wildtype siblings ( $\mathrm{n}=20$ nerves, 11 larvae) and ext/3 $/-{ }^{-}$larvae $(\mathrm{n}=30$ nerves, 16 larvae) and $(K)$ robo2 wildtype siblings $(n=10$ nerves, 5 larvae) and robo2 $-/-$ larvae $(n=17$ nerves, 9 larvae). Nerves were scored by counting the number of discrete fascicles discernible 50 um from the MEP (yellow arrowhead in A). Genotypes were compared using two-tailed t-tests (for ext/3: Precut, $p=0.6875 ; 48 \mathrm{hpt}$, $p=0.9167$ for robo2: Precut $p=0.6009 ; 48 \mathrm{hpt}, \mathrm{p}=0.1003$ Angle of ventral nerve extension at 5 dpf and $48 \mathrm{hpt}$ in $(F)$ ext/3 wildtype siblings $(n=10$ nerves, 5 larvae) and ext $/ 3^{-/-}$larvae $\left(n=17\right.$ nerves, 9 larvae) and $(L)$ robo2 siblings $\left(n=26\right.$ nerves, 10 larvae) and robo2 ${ }^{-/-}$larvae $(n=17$ nerves, 7 larvae). Extension angle was calculated as the difference between the angle of the nerve between 50um from the MEP (yellow arrowhead in A) and 100um from MEP (blue arrowhead in A). Each dot represents one nerve. Genotypes were compared using two-tailed t-tests (for ext/3: Precut, $p=0.3403 ; 48 \mathrm{hpt}, \mathrm{p}=0.1900 ;$ for robo2: Precut $p=0.9424 ; 48 \mathrm{hpt}, \mathrm{p}=0.8731$ ). 


\section{A Errors formed (8-20hpt)}

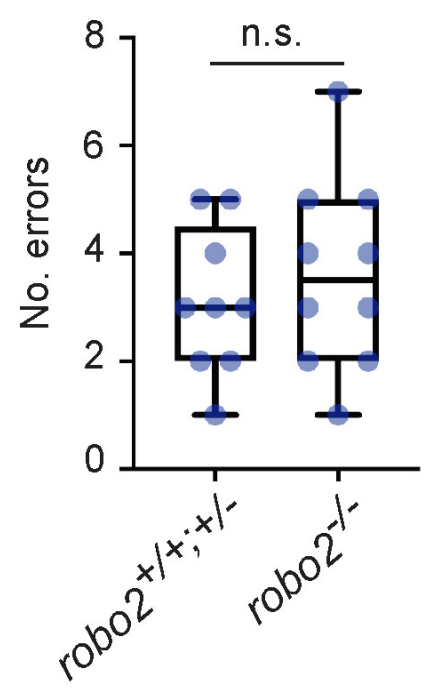

B

Speed of error movements (8-20hpt)

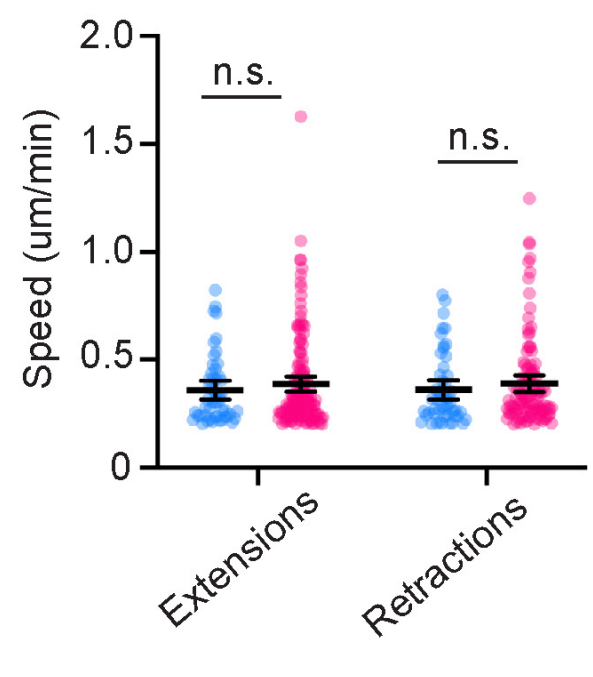

robo $2^{+/+;+/-}$

- robo $2^{-/-}$

Fig. S3. Error formation and regenerating fascicle growth dynamics are unaffected in robo2 mutant. (A) Number of errors formed 8-20hpt in wildtype siblings (robo2 $^{+/+;+/-}, \mathrm{n}=9$ nerves) and robo2 ${ }^{-/-}$larvae $(\mathrm{n}=10$ nerves). Each dot represents one nerve. Ranks between genotypes were compared using two-tailed Mann Whitney test $(p=0.6238)(B)$ Error extension and retraction speed calculated as the absolute value of error movement velocity in um/min. Extension and retraction events were examined in $10 \mathrm{~min}$ intervals and classified as extensions when there was a $\geq 1$ um increase in error length measured from the motor exit point (MEP); movements were classified as retractions when $\geq 1$ um decrease in error length measured from the MEP. Each dot represents one extension or retraction event (for siblings $n=23$ errors underwent 51 extension events and 51 retraction events; for robo2 ${ }^{-/-}, \mathrm{n}=34$ errors underwent 139 extension events and 109 retraction events). Line, mean; error bars, 95\% confidence intervals. Genotypes were compared using two-tailed t-tests (for extensions, $p=0.3849$; for retractions, $p=0.3853$ ). n.s. denotes "not significant." 

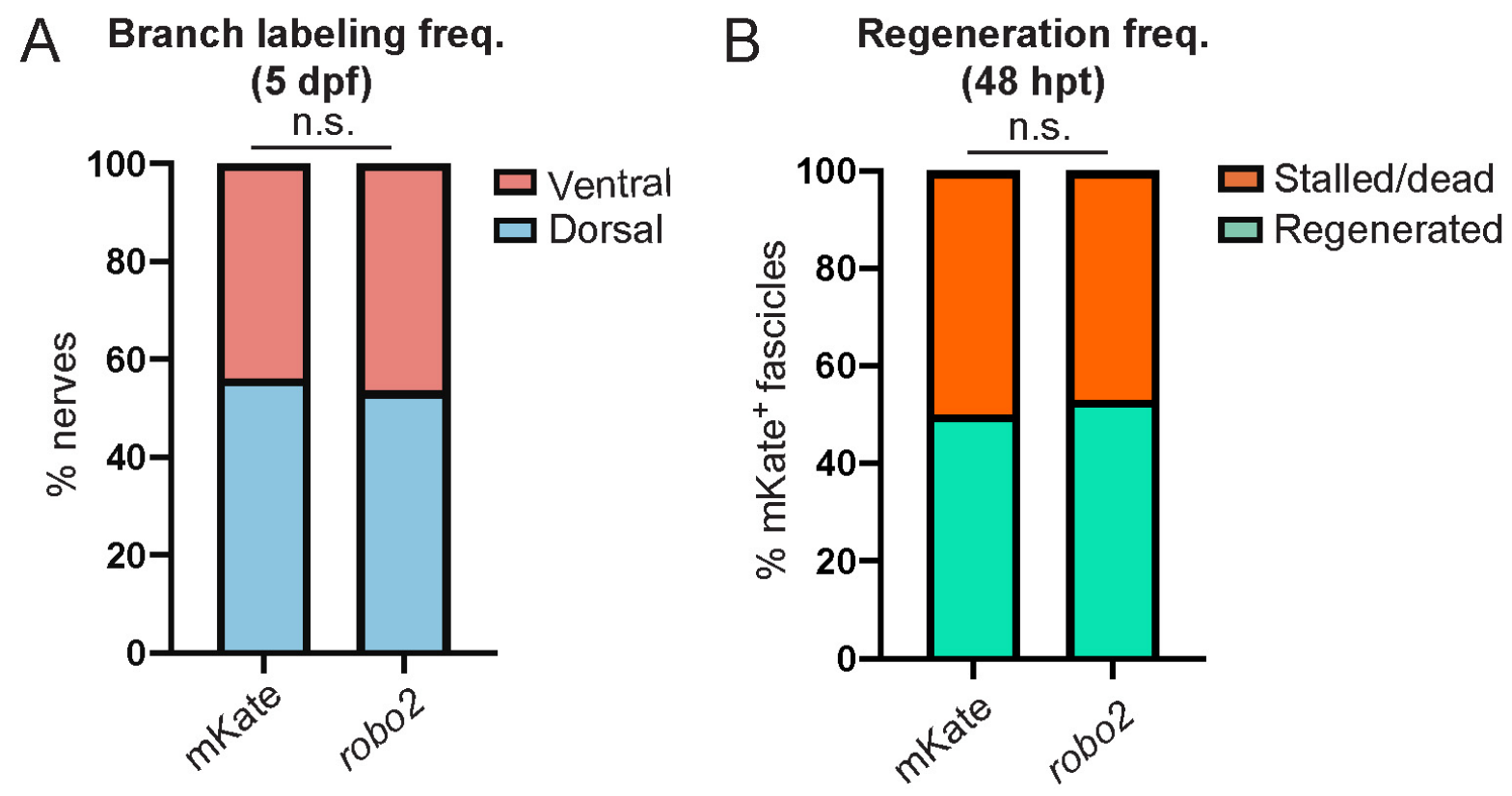

Fig. S4. Robo2 does not affect developmental branch-selectivity or regeneration capacity of spinal motor axons. (A) Graph of developmental branch-selectivity of sparsely labeled fascicles in $T g(h b 9: G F P)$ larvae, showing the percent of spinal motor nerves in which any fascicles expressing $T g(m n \times 1: m K a t e ; ~ m n x 1: m K a t e)$ (mKate in graph) or $\mathrm{Tg}(\mathrm{mn} \times 1: \mathrm{mKate} ; \mathrm{mnx1}$ :robo2) (robo2 in graph) developed along the dorsal nerve branch (dorsal) or along the ventral branch exclusively (ventral) at $5 \mathrm{dpf}$ (for $\operatorname{Tg}$ (mnx1:mKate; $m n \times 1: m K a t e), \mathrm{n}=41$ nerves; for $\operatorname{Tg}(m n \times 1: m K a t e ; m n \times 1: r o b o 2), \mathrm{n}=41$ nerves). Two-tailed Fischer's exact test was used to compare proportions of nerve counts in each category $(p=0.999)$. (B) Graph of regeneration frequency of fascicles showing the percent of spinal motor nerves in which fascicles expressing $\mathrm{Tg}$ (mnx1:mKate; $m n \times 1: m K a t e)$ (mKate in graph) or $\mathrm{Tg}(\mathrm{mn} \times 1: \mathrm{mKate}$; $m n \times 1:$ robo2) (robo2 in graph) regenerated at least 50um along either nerve branch (Regenerated) or failed to regenerate (Stalled/dead) at 48 hpt (for $\operatorname{Tg}(m n \times 1$ :mKate; $m n \times 1: m K a t e), n=26$ nerves; for $\operatorname{Tg}(m n \times 1$ : $m K$ Kate; $m n \times 1$ :robo2), $\mathrm{n}=32$ nerves). Two-tailed Fischer's exact test was used to compare proportions of nerve counts in each category $(p=0.999)$. n.s. denotes "not significant." 

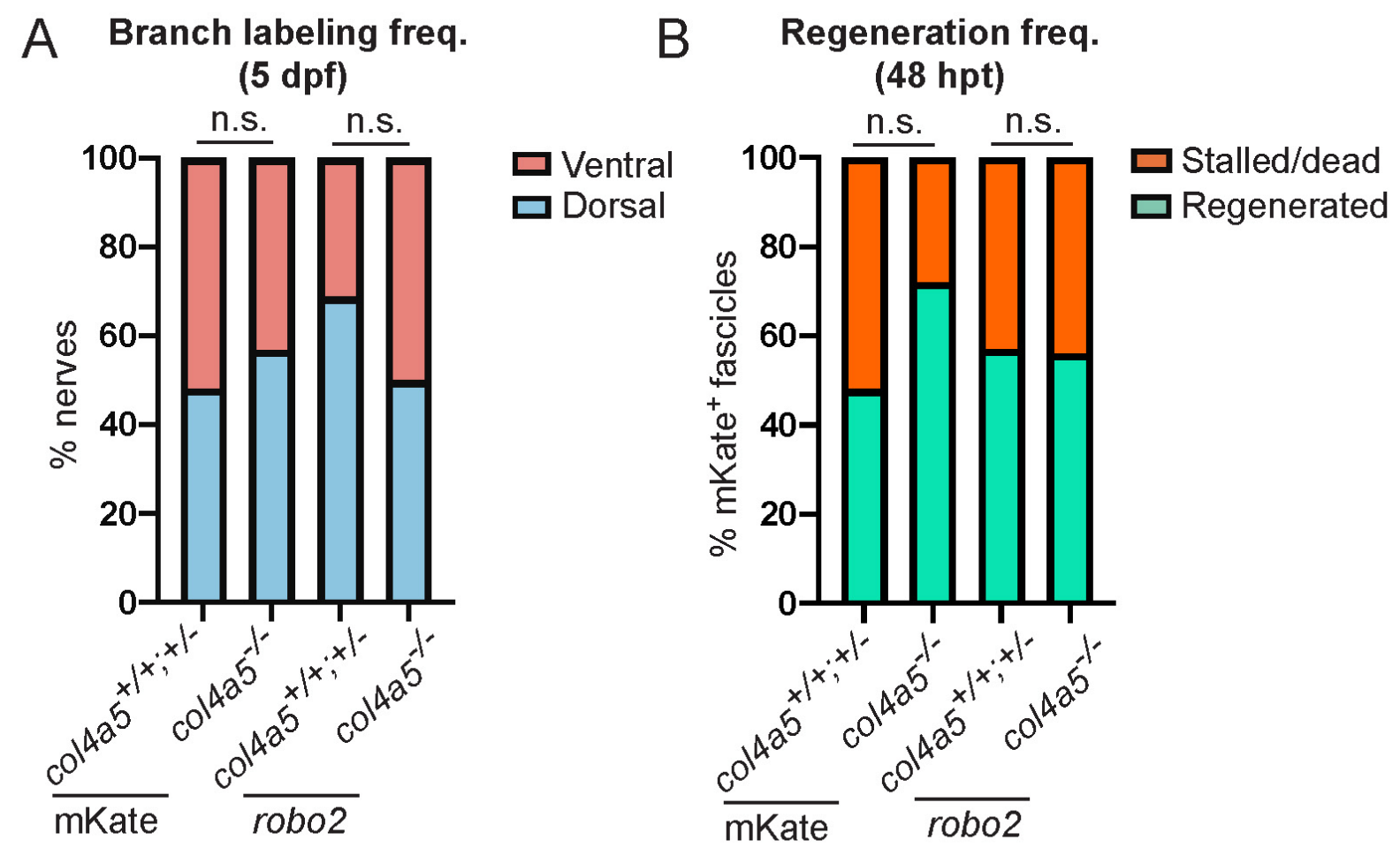

Fig. S5. Robo2 does not affect developmental branch-selectivity or regeneration capacity of spinal motor axons in col4a5 $+/+;+/-\quad$ wildtype siblings) and col4a5 $/$. . (A) Graph of developmental branch-selectivity of sparsely labeled fascicles in in col4a5 $5^{+/++/-}$(wildtype siblings) and col4a5 $5^{-/-} \mathrm{Tg}$ (hb9:GFP) larvae, showing the percent of spinal motor nerves in which any fascicles expressing $\operatorname{Tg}(m n x 1: m K a t e ; m n x 1: m K a t e)$ (mKate in graph) or Tg(mnx1:mKate; $m n x 1:$ robo2) (robo2 in graph) developed along the dorsal nerve branch (dorsal) or along the ventral branch exclusively (ventral) at $5 \mathrm{dpf}$ (for wildtype siblings: $T g(m n \times 1: m K a t e ; m n \times 1: m K a t e), \mathrm{n}=$ 89 nerves; for $\operatorname{Tg}(m n x 1: m K a t e ; m n x 1: r o b o 2), \mathrm{n}=80$ nerves; for col4a5 $/$ - larvae $\operatorname{Tg}(m n x 1: m K a t e ; m n x 1: m K a t e), \mathrm{n}=48 \mathrm{nerves} ;$ for $\operatorname{Tg}(m n x 1: m K a t e ; m n x 1: r o b o 2), \mathrm{n}=26$ nerves). Two-tailed Fischer's exact test was used to compare proportions of nerve counts in each category (for siblings, $p=0.2831 ;$ for col4a5 $/-1$ larvae, $p=0.1364)$. $(B)$ Graph of regeneration frequency of fascicles showing the percent of spinal motor nerves in col4a5 $5^{+/+; /-}$(wildtype siblings) and col4a5 $/-$Tg(hb9:GFP) larvae in which fascicles expressing $\operatorname{Tg}(m n x 1: m K a t e ; m n x 1: m K a t e)$ (mKate in graph) or $\operatorname{Tg}(m n \times 1: m K a t e ; m n x 1: r o b o 2)$ (robo2 in graph) regenerated at least 50um along either nerve branch (Regenerated) or failed to regenerate (Stalled/dead) at $48 \mathrm{hpt}$ (for wildtype siblings: $\operatorname{Tg}(m n x 1: m K a t e ; m n x 1: m K a t e), \mathrm{n}=24 \mathrm{nerves;}$ for $T g(m n x 1: m K a t e ; m n x 1: r o b o 2), \mathrm{n}=21$

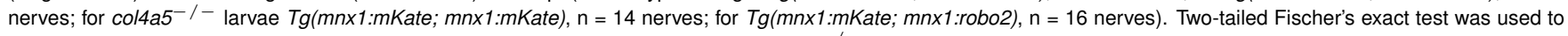
compare proportions of nerve counts in each category (for siblings, $p=0.3661$; for col4a5 $-/$ larvae, $p=0.999$ ). n.s. denotes "not significant." 
bioRxiv preprint doi: https://doi.org/10.1101/2020.12.07.406942; this version posted December 7, 2020. The copyright holder for this preprint (which was not certified by peer review) is the author/funder. All rights reserved. No reuse allowed without permission.

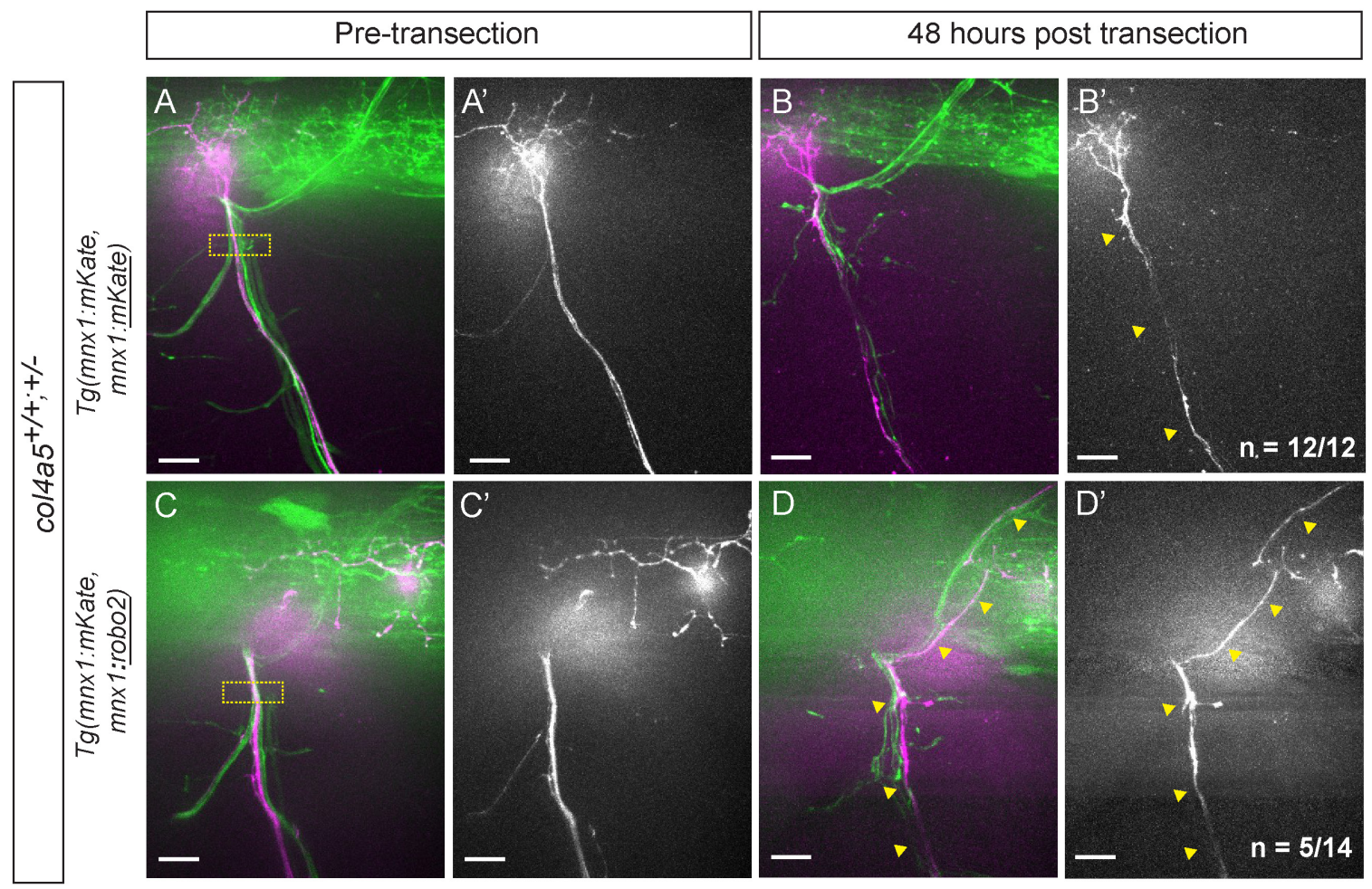

Fig. S6. robo2 is sufficient to promote dorsal branch-selection by regenerating axons in col4a $5^{+/+;+/-}$larvae (wildtype siblings). Representative images of $\operatorname{Tg}\left(\right.$ hb9:GFP) (green) nerves in col4a $5^{+/-}$(wildtype sibling) larvae with fascicles expressing transient $\operatorname{Tg}(m n \times 1: m K a t e, ~ m n \times 1: m K a t e)$ (A-B) or $T g(m n \times 1: m K a t e, ~ m n \times 1: r o b o 2)$ (C-D) (magenta) in small subsets of ventrally projecting motor axons. Merged GFP and mKate images shown at $5 \mathrm{dpf}(\mathrm{A}, \mathrm{C})$ and $48 \mathrm{hpt}(\mathrm{B}, \mathrm{D})$. mKate channel is shown alone at $5 \mathrm{dpf}\left(\mathbf{A}^{\prime}, \mathbf{C}^{\prime}\right)$ and 48hpt (B', textbfD'). In larvae expressing $T g(m n \times 1: m K a t e, m n x 1: m K a t e), n=12 / 12$ nerves had only ventral regrowth of mKate ${ }^{+}$fascicles, like in the example shown. In larvae expressing $T g$ (mnx1:mKate, $m n \times 1$ :robo2), $n=5 / 14$ nerves had dorsal regrowth of mKate ${ }^{+}$fascicles, like in the example shown. Proportions of ventral regrowth were compared between conditions using one-tailed Fischer's exact test $(p=0.0304)$. Images were processed as described in Materials and Methods. Dashed yellow boxes, transection site. Scale bars, 10um. 


\section{Legends for Supplementary Movies}

Movie S1. Dorsal nerve fascicle regeneration dynamics in wildtype sibling, related to Figure 3. Representative movie of regenerating axons of dorsal nerve in robo $2^{+/-}$larvae imaged in vivo using $T g(i s l 1: G F P)$. The movie begins 8 hpt, and images were taken every 10 minutes, as indicated by time counter (white, bottom right), for 12 hours. Cumulative error count (magenta, bottom right) is written as a fraction of [number errors corrected/over number errors formed]. Error fascicle movements are marked with magenta arrowheads. Movements of fascicle growing along the dorsal path are marked with green arrowheads. Images were processed as described in Materials and Methods. Scale bar is 10um.

Movie S2. Dorsal nerve fascicle regeneration dynamics in robo2 mutant, related to Figure 3. Representative movie of regenerating axons of dorsal nerve in robo $2^{-/-}$larvae imaged in vivo using $\operatorname{Tg}($ isll:GFP). The movie begins 8 hpt, and images were taken every 10 minutes, as indicated by time counter (white, bottom right), for 12 hours. Cumulative error count (magenta, bottom right) is written as a fraction of [number errors corrected/over number errors formed]. Error fascicle movements are marked with magenta arrowheads. Movements of fascicle growing along the dorsal path are marked with green arrowheads. Images were processed as described in Materials and Methods. Scale bar is 10um. 